\title{
Investigating Nonlinearity: A Note on the Estimation of Hamilton's Random Field Regression Model ${ }^{*}$
}

\author{
D. Bond, M.J. Harrison and E.J. O'Brien \\ University of Ulster and Trinity College Dublin
}

April 6, 2005

\begin{abstract}
This is a revised and extended version of the authors' 2003 Trinity Economic Paper. It describes Hamilton's (2001) approach to nonlinear econometric modelling and some of the methods of nonlinear optimization, as before, but adds significantly to the investigation of Hamilton's Gauss program for the implementation of his methodology. Specifically, it reports on the performance of this program using data relating to Hamilton's US Phillips curve example, the use of two versions of the Gauss software and a range of numerical optimization options. It also examines the impact of changes in initial parameter estimates, the use of algorithm switching strategies, and the effects of changes in the sample data on the results produced by Hamilton's procedure. The new results presented suggest some further clear conclusions that will be of value to those using Hamilton's method.
\end{abstract}

J.E.L. Classification: C13, C51, C61

Keywords: nonlinearity, numerical optimization, US Phillips curve.

*A modified version of this paper is forthcoming in the September 2005 issue of Studies in Nonlinear Dynamics and Econometrics. The authors are grateful to B. Mizrach, Editor of SNDE, D.A. Belsley, C.M. Dahl, and J.D. Hamilton for their assistance, and to an anonymous referee for some helpful comments. E.J. O'Brien, who is a Government of Ireland Scholar, also wishes to thank the Irish Council for the Humanities and Social Sciences for its generous funding. 


\title{
Investigating Nonlinearity: A Note on the Estimation of Hamilton's Random Field Regression Model
}

\author{
by \\ D. Bond, M.J. Harrison and E.J. O'Brien
}

\section{Introduction}

In an important recent paper, Hamilton (2001) proposed an approach to nonlinear modelling of economic relationships that provides a single flexible parametric framework for testing for nonlinearity, drawing inference about the form of nonlinearity, and assessing the adequacy of the description of nonlinearity provided by specific models. Following Wecker and Ansley (1983), the approach treats functional form as the outcome of a latent stochastic process that is part of the data-generating process; that is, the conditional expectation function associated with a regression model is thought of as being generated randomly prior to the generation of the data. This latent process is modelled using a new Gaussian random field concept that generalizes Brownian motion to $k$ dimensions, and the parameters of the process are estimated by maximum likelihood. The method is a good deal more than an exploratory data or data-smoothing device. From the practicing economist's viewpoint, its importance lies in the valuable insights it can provide for model construction and the resulting enhancement of the forecasting ability of economic models.

However, the new methodology has been little used to date and its full potential remains to be established. As Hamilton (2001, p. 552) points out, its usefulness for particular sample sizes and nonlinearities is a matter for empirical investigation. Yet, citing his own three examples and the Monte Carlo studies by Dahl (1998), he suggests that the method holds much promise. We very much agree.

The aims of the present paper are modest and the focus intentionally narrow. The main purpose is to address a number of practical issues that arise when using the Hamilton approach. The first of these concerns computation: we report on our experience with Hamilton's software to implement the method. The program is written in Gauss and can be freely downloaded from http://weber.ucsd.edu/ jhamilto. It appears that the numerical optimization involved is not an entirely straightforward matter, either when using Hamilton's own dataset or our alternative samples. It should be stressed at the outset that this is a result of difficulties with numerical optimization and not the Hamilton method itself. Thus while the Hamilton case 
study is our primary focus, this finding may have more general relevance for procedures that employ similar optimization techniques. The second issue concerns the sensitivity of the method to changes in data. Our experiments suggest that minor data changes can have implications for computation and big effects on the results. Another aim, given the length and difficulty of the original paper, is to provide a concise and reasonably accessible account of Hamilton's methodology for non-specialist practitioners, though the nature of the subject is such that it is not possible to avoid technicalities.

The structure of the paper is as follows. Section 2 describes the new approach to modelling nonlinearity; sections 3 and 4 report on computational and datasensitivity matters, respectively; and section 5 contains a brief summary and conclusion.

\section{Hamilton's Method of Parametric Flexible Nonlinear Inference}

To facilitate cross-reference to the original paper, the notation used in this section is similar to that used by Hamilton (2001).

\subsection{The model}

Our interest is in the nonlinear regression denoted by

$$
y_{t}=\mu\left(\mathbf{x}_{t}\right)+\varepsilon_{t}, \quad t=1,2, \ldots, T,
$$

where $y_{t}$ is a scalar, $\mathbf{x}_{t}=\left[x_{i t}\right]$ is a $k$-vector of observations on the explanatory variables at time $t, \varepsilon_{t}$ is a stochastic disturbance with zero mean and constant variance, independent of lagged values of $\mathbf{x}_{t}$ and $y_{t}$, and $\mu(\mathbf{x})$ denotes the conditional expectation function $E(y \mid \mathbf{x})$. The nature of $\mu(\mathbf{x})$ is fundamental to Hamilton's approach and is considered to be determined as

$$
\mu(\mathbf{x})=\alpha_{0}+\boldsymbol{\alpha}^{\prime} \mathbf{x}+\lambda m(\mathbf{g} \odot \mathbf{x})
$$

where $\alpha_{0}$ and $\lambda$ are scalar parameters, $\boldsymbol{\alpha}=\left[\alpha_{i}\right]$ and $\mathbf{g}=\left[g_{i}\right]$ are $k$-vectors of parameters, $m(\cdot)$ is a realization of a stochastic process called a random field, and $\odot$ denotes element-by-element multiplication. The realization $m(\cdot)$, hence $\mu(\mathbf{x})$, is assumed to be generated by nature prior to and independently of all of the observations. Given this fixed $\mu(\mathbf{x})$, the values for $\varepsilon_{t}$ and $\mathbf{x}_{t}$ are then generated and $y_{t}$, is determined according to the regression (2.1). 
The interpretation of the parameters in (2.2) is vitally important for the application of Hamilton's method. In particular, the scalars $\lambda$ and $g_{i}, i=1,2, \ldots, k$, characterize the relationship between $m(\cdot)$ and the conditional expectation function $\mu\left(x_{1}, x_{2}, \ldots, x_{k}\right)$. Specifically, $\lambda$ is a measure of the overall 'weight' of the process $m(\cdot)$ in the conditional expectation, while the magnitudes of the $g_{i}$ indicate the degree of nonlinearity associated with their respective $x_{i}$. Thus $\lambda=0$ indicates that $m(\cdot)$ makes no contribution and the conditional expectation is linear, in which case (2.1) is the familiar general linear model. Similarly, $g_{i}=0$ implies that the conditional expectation is linear in $x_{i}$, while $g_{i} \neq 0$ signifies that it is nonlinear in $x_{i}$. If all of the $g_{i} \rightarrow 0$, the contribution of $m(\cdot)$ to the conditional expectation, hence to $y_{t}$, becomes indistinguishable from that of $\alpha_{0}$; if all of the $g_{i} \rightarrow \infty$, the contribution to $y_{t}$ is indistinguishable from that of $\varepsilon_{t}$. The interpretation associated with $\alpha_{0}$ and the $\alpha_{i}$ is the standard one.

The key component in (2.2), on which the interpretation of the $g_{i}$ depends, is the random realization $m(\cdot)$, whose nature and role require explanation before the practical matters of estimation and testing are considered. First, consider a uniform orthogonal grid in $\Re^{k}$, bounded in the direction of each of the $k$ standard basis vectors or Cartesian co-ordinates by some lower value $a_{j}$ and some upper value $b_{j}, j=1,2, \ldots, k$. By uniform we mean that the intervals defined by the grid are of equal length in the direction of each of the $k$ co-ordinates, and the number of intervals in each direction is the same. Note that this does not imply that the intervals in different directions have to be the same length unless the $a_{j}$ are equal and the $b_{j}$ are equal $\forall j$. Let the set of all nodes in the grid be $A_{N}$, where $N-1$ is the number of grid intervals in each direction and $N^{k}$ is therefore the number of distinct points in $A_{N}$. For each point $\mathbf{x} \in A_{N}$, let $e(\mathbf{x}) \sim \mathbb{N}(0,1)$ and be independent of $e(\mathbf{z})$ for all $\mathbf{x} \neq \mathbf{z}$; let $B_{N}(\mathbf{x})=\left\{\mathbf{z} \in A_{N}:(\mathbf{x}-\mathbf{z})^{\prime}(\mathbf{x}-\mathbf{z}) \leq 1\right\}$, i.e., the set of all points in $A_{N}$ whose distance from $\mathbf{x}$ is less than or equal to unity; and let $n_{N}(\mathbf{x})$ denote the number of points in $B_{N}(\mathbf{x})$. Hamilton $(2001$, p.540) then defines the scalar process $m_{N}(\mathbf{x})$ as

$$
m_{N}(\mathbf{x})=\left[n_{N}(\mathbf{x})\right]^{-\frac{1}{2}} \sum_{\mathbf{z} \in B_{N}(\mathbf{x})} e(\mathbf{z}) .
$$

This process is illustrated for $k=2, a_{1}=a_{2}=0, b_{1}=5, b_{2}=3$, and equal interval lengths in Hamilton (2001, p.541), so that the number of intervals in each direction is not the same, as required by the definition of $A_{N}$. Taking the limit of (2.3) as the grid partition becomes finer, i.e., as $N \rightarrow \infty$ and the interval length in each direction of the grid tends to zero, we have the notion of a continuous 
scalar-valued $k$-dimensional random field. The stochastic nature of this is such that for any $\mathbf{x} \in A_{N}, m(\mathbf{x}) \sim \mathbb{N}(0,1)$. The similarity to standard Brownian motion is apparent.

For arbitrary points $\mathbf{x}$ and $\mathbf{z}$ in $\Re^{k}$, the correlation between $m(\mathbf{x})$ and $m(\mathbf{z})$ is zero if the distance between $\mathbf{x}$ and $\mathbf{z}$ is greater than 2 . If this distance is not greater than 2 , it can be shown, though the proofs are difficult, that

$$
H_{k}(h)=\operatorname{Cov}_{k}(m(\mathbf{x}), m(\mathbf{z}))=\frac{G_{k-1}(h, 1)}{G_{k-1}(0,1)}
$$

where $G_{k-1}(h, 1)=-\frac{h}{k}(1-h)^{\frac{k-1}{2}}+\frac{k-1}{k} G_{k-3}(h, 1), h$ is one-half the distance between $\mathbf{x}$ and $\mathbf{z}, k=2,3, \ldots$ and the initial values are $G_{0}(h, 1)=1-h$ and $G_{1}(h, 1)=\frac{\pi}{4}-\frac{1}{2} h\left(1-h^{2}\right)^{\frac{1}{2}}-\frac{1}{2} \sin (h)$. See Lemma 2.1 and Theorem 2.2 in Hamilton (2001, p. 541), and note that the details relating to Equation (2.4) are expressed slightly differently than in Hamilton's lemma and theorem. Equation (2.4) can be calculated recursively, but fortunately its values for $k=1$ to 5 inclusive are provided in Table I of Hamilton (2001, p. 542). It is this covariance that provides the means by which the $g_{i}$ govern the curvature of $\mu(\mathbf{x})$ in $(2.2)$; see the illustrative case of $k=1$ in Hamilton (2001, p. 540).

\subsection{Estimation}

Assuming normality of the $\varepsilon_{t}$, it follows from (2.1), (2.2) and (2.4) that

$$
\mathbf{y} \sim \mathbb{N}\left(\mathbf{X} \boldsymbol{\beta}, \mathbf{C}+\sigma^{2} \mathbf{I}_{T}\right)
$$

where $\mathbf{y}$ is the $T$-vector of observations on the dependent variable in (1), $\mathbf{X}$ is the $T \times(k+1)$ matrix of observations on the $k$ explanatory variables and a column of ones associated with the intercept, $\boldsymbol{\beta}=\left[\begin{array}{ll}\alpha_{0} & \boldsymbol{\alpha}^{\prime}\end{array}\right]^{\prime}$ is the $(k+1)$ vector of parameters of the linear component of the conditional expectation, $\mathbf{C}=\left[\lambda^{2} H_{k}\left(h_{t s}\right)\right]$ is a $T \times T$ variance-covariance matrix whose typical element is $\lambda^{2} \operatorname{Cov}_{k}\left(m\left(\mathbf{g} \odot \mathbf{x}_{t}\right), m\left(\mathbf{g} \odot \mathbf{x}_{s}\right)\right)$, and $h_{t s}$ is one-half the distance between $\mathbf{g} \odot \mathbf{x}_{t}$ and $\mathbf{g} \odot \mathbf{x}_{s}$. The likelihood function follows straightforwardly from (2.5) as

$\ln f\left(\mathbf{y} ; \boldsymbol{\beta}, \mathbf{g}, \lambda, \sigma^{2}\right)=-\frac{T}{2} \ln (2 \pi)-\frac{1}{2} \ln \left|\mathbf{C}+\sigma^{2} \mathbf{I}_{T}\right|-\frac{1}{2}(\mathbf{y}-\mathbf{X} \boldsymbol{\beta})^{\prime}\left(\mathbf{C}+\sigma^{2} \mathbf{I}_{T}\right)^{-1}(\mathbf{y}-\mathbf{X} \boldsymbol{\beta})$.

Maximum likelihood provides the basis for inference concerning the parameters $\boldsymbol{\beta}, \mathbf{g}, \lambda$ and $\sigma^{2}$; and as Hamilton shows, the procedure is valid for regressors that 
are deterministic or lagged values of the dependent variable. However, in the interests of simplifying the calculations, (2.6) is re-written. Defining $\zeta=\frac{\lambda}{\sigma}$, letting $\boldsymbol{\psi}=\left[\begin{array}{ll}\boldsymbol{\beta}^{\prime} & \sigma^{2}\end{array}\right]^{\prime}$ be the $(k+2)$-vector of parameters of the linear part of the model and $\boldsymbol{\theta}=\left[\mathbf{g}^{\prime} \zeta\right]^{\prime}$ be the $(k+1)$-vector of parameters of the nonlinear component, and setting $\mathbf{W}(\mathbf{X} ; \boldsymbol{\theta})=\zeta^{2} \mathbf{C}^{*}+\mathbf{I}_{T}$, where $\mathbf{C}^{*}=\lambda^{-2} \mathbf{C}$, the right-hand side of (2.6) can be written as

$$
-\frac{T}{2} \ln (2 \pi)-\frac{T}{2} \ln \sigma^{2}-\frac{1}{2} \ln |\mathbf{W}(\mathbf{X} ; \boldsymbol{\theta})|-\frac{1}{2 \sigma^{2}}(\mathbf{y}-\mathbf{X} \boldsymbol{\beta})^{\prime} \mathbf{W}(\mathbf{X} ; \boldsymbol{\theta})^{-1}(\mathbf{y}-\mathbf{X} \boldsymbol{\beta}) .
$$

The values of the elements of $\boldsymbol{\psi}$ that maximize (2.7) for given $\boldsymbol{\theta}$ can then be calculated analytically as

$$
\begin{aligned}
\widetilde{\boldsymbol{\beta}}(\boldsymbol{\theta}) & =\left[\mathbf{X}^{\prime} \mathbf{W}(\mathbf{X} ; \boldsymbol{\theta})^{-1} \mathbf{X}\right]^{-1} \mathbf{X}^{\prime} \mathbf{W}(\mathbf{X} ; \boldsymbol{\theta})^{-1} \mathbf{y} \\
\widetilde{\sigma}^{2}(\boldsymbol{\theta}) & =\frac{1}{T}[\mathbf{y}-\mathbf{X} \widetilde{\boldsymbol{\beta}}(\boldsymbol{\theta})]^{\prime} \mathbf{W}(\mathbf{X} ; \boldsymbol{\theta})^{-1}[\mathbf{y}-\mathbf{X} \widetilde{\boldsymbol{\beta}}(\boldsymbol{\theta})] .
\end{aligned}
$$

Thus (2.7) may be concentrated as

$$
\phi(\boldsymbol{\theta} ; \mathbf{y}, \mathbf{X})=-\frac{T}{2} \ln (2 \pi)-\frac{T}{2} \ln \widetilde{\sigma}^{2}(\boldsymbol{\theta})-\frac{1}{2} \ln |\mathbf{W}(\mathbf{X} ; \boldsymbol{\theta})|-\frac{T}{2} .
$$

Hence numerical maximization of (2.10) gives the maximum likelihood estimate of $\boldsymbol{\theta}$, which, via (2.8) and (2.9), yields the estimate of $\boldsymbol{\psi}$.

\subsection{Testing for nonlinearity}

The form of the model used in Hamilton's approach suggests that a simple method of testing for nonlinearity is to check if $\lambda$, or $\lambda^{2}$, is zero or not. Hamilton shows that if $\lambda^{2}=0$, and the nonlinear model is estimated, then for fixed $\mathbf{g}$, the maximum likelihood estimator $\widetilde{\lambda}^{2}$ is consistent for the true value of zero and asymptotically normal. Thus a test based on the use of standard normal tables is suggested. However, given the maximum likelihood approach to estimation and the linearity of (2.1) under the null hypothesis that $\lambda^{2}=0$, an obvious and perhaps more appealing way of testing is to use the Lagrange multiplier principle, which requires only a simple linear regression to be estimated. Under the assumption of normality, Hamilton derives the appropriate score vector of first derivatives and the associated information matrix and proposes a form of LM test for practical application. The procedure is as follows: 
- Set $g_{i}=\frac{2}{\sqrt{k s_{i}^{2}}}$, where $s_{i}^{2}$ is the variance of explanatory variable $x_{i}$, excluding the constant term whose variance is zero. This $g_{i}$ is approximately the mean of the lognormal Bayesian prior used by Hamilton (2001, Section 5) for $\theta$.

- Calculate the $T \times T$ matrix, $\mathbf{H}$, whose typical element is $H_{k}\left(\frac{1}{2}\left\|\mathbf{g} \odot \mathbf{x}_{t}-\mathbf{g} \odot \mathbf{x}_{s}\right\|\right)$, i.e., the function $H_{k}\left(h_{t s}\right)$ defined in (2.4) and (2.5).

- Use OLS to estimate the standard linear regression $\mathbf{y}=\mathbf{X} \boldsymbol{\beta}+\boldsymbol{\epsilon}$ and obtain the usual residuals, $\widehat{\boldsymbol{\epsilon}}$, and standard error of estimate, $\widehat{\sigma}=(T-k-1)^{-\frac{1}{2} \sqrt{\widehat{\boldsymbol{\epsilon}}^{\prime} \widehat{\boldsymbol{\epsilon}}}}$.

- Finally, compute the statistic

$$
\varkappa^{2}=\frac{\left[\widehat{\boldsymbol{\epsilon}}^{\prime} \mathbf{H} \widehat{\boldsymbol{\epsilon}}-\widehat{\sigma}^{2} \operatorname{tr}(\mathbf{M H})\right]^{2}}{\widehat{\sigma}^{4}\left\{2 \operatorname{tr}\left(\left[\mathbf{M H M}-(T-k-1)^{-1} \mathbf{M} \operatorname{tr}(\mathbf{M H})\right]^{2}\right)\right\}},
$$

where $\mathbf{M}=\mathbf{I}_{T}-\mathbf{X}\left(\mathbf{X}^{\prime} \mathbf{X}\right)^{-1} \mathbf{X}^{\prime}$ is the familiar symmetric idempotent matrix.

As $\varkappa^{2} \stackrel{A}{\sim} \chi_{1}^{2}$ under the null hypothesis, linearity $\left(\lambda^{2}=0\right)$ would be rejected if $\varkappa^{2}$ exceeded the critical value, $\chi_{1, \alpha}^{2}$, for the chosen level of significance, $\alpha$. Otherwise the null of linearity would not be rejected. For example, at the 5 per cent significance level, the null would be rejected if $\varkappa^{2}>3.84$. In this case the alternative nonlinear specification given by (2.1) and (2.2) would be preferred. The identification of a specific form of nonlinearity is greatly aided by the estimate of the conditional expectation $\mu\left(\mathbf{x}_{t}\right)$ and, specifically, the $\widetilde{g}_{i}$ and $\widetilde{\zeta}$. The matter is explained in Hamilton (2001, Section 5) and illustrated in the three examples in his Section 7; note the role of his Equation (5.17). However, this is not pursued in the present paper, which concentrates on the nonlinear estimation per se.

\section{Computational Issues}

The implementation of Hamilton's methodology is straightforward, in principle, given the on-line availability of Hamilton's software. In practice, however, difficulties may await the unwary. These difficulties relate to the nonlinear optimization algorithms in the OPTMUM procedure in Gauss, which is at the heart of Hamilton's program. Indeed, in our original attempts to run the program using Gauss 5, the optimization procedure failed completely and no nonlinear estimates were obtained. It was this experience that motivated the research for the present paper. 
The methods of nonlinear optimization are familiar to most econometricians, and the particular algorithms available in Gauss may be familiar to some Gauss users. However, most economists probably do not share this familiarity. Therefore, to assist with the understanding of the material in this and the following section, a brief description of numerical optimization and the relevant algorithms is provided in the following subsection. Further details on numerical optimization may be found in the texts by Brent (1973), Greene (2003), Murray (1972) and the Gauss reference manual Optimization, Aptech Systems, Inc., 2001, especially chapters 2 and 3 .

\subsection{Nonlinear optimization}

The OPTMUM procedure in Gauss maximizes the function $(2.10), \phi(\boldsymbol{\theta} ; \mathbf{y}, \mathbf{X})$, by minimizing the negative of the function with respect to its vector of parameters $\boldsymbol{\theta}$. Given the derivatives of this objective function with respect to $\boldsymbol{\theta}$, i.e., the gradient vector, which it computes numerically, and initial values for $\boldsymbol{\theta}$, OPTMUM proceeds iteratively, computing a direction, $\mathbf{d}$, and a step length, $s$, at each iteration. The quantity $s \mathbf{d}$ is a vector of values that is added to the current estimate of $\boldsymbol{\theta}$, and therefore has the same dimension as $\boldsymbol{\theta}$, and $s$ is a scalar. Thus, given a value for $\mathbf{d}$, the current estimate, $\widetilde{\boldsymbol{\theta}}$, is updated as

$$
\widetilde{\boldsymbol{\theta}}_{+}=\widetilde{\boldsymbol{\theta}}+s \mathbf{d}
$$

hence $s$ may be interpreted as changing the rate of descent of the objective function in the given direction. We describe in turn how $\mathbf{d}$ and $s$ are computed, concentrating on the former.

Defining $\mathbb{G}$ to be the $(k+1)$ gradient vector and $\mathbb{H}$ to be a $(k+1) \times(k+1)$ symmetric matrix, a standard method of calculating $\mathbf{d}$ is as

$$
\mathbf{d}=\mathbb{H}^{-1} \mathbb{G} \text {. }
$$

However, as numerical matrix inversion may be a risky process, the OPTMUM procedure can avoid it by computing $\mathbf{d}$ as the solution of the equation

$$
\mathbb{H} \mathbf{d}=\mathbb{G},
$$

which is thought to be numerically more reliable. While $\mathbb{G}$ is calculated in a standard manner, $\mathbb{H}$ may be calculated in different ways depending on which algorithm is selected. Several approaches are available in OPTMUM. 
The steepest descent algorithm simply sets $\mathbb{H}=\mathbf{I}_{(k+1)}$. While this is computationally undemanding and therefore attractive, the descent may be slow and require many iterations before convergence.

The PRCG or Polak and Ribiere (1969) conjugate gradient method is a development of the steepest descent method that also uses only the gradient but updates the direction as

$$
\mathbf{d}_{+}=\mathbb{G}_{+}+r \mathbf{d}, \text { where } r=\frac{\left(\mathbb{G}_{+}-\mathbb{G}\right)^{\prime} \mathbb{G}_{+}}{\mathbb{G}^{\prime} \mathbb{G}} .
$$

There are several more complex methods. The Newton algorithm equates $\mathbb{H}$ to the Hessian of the objective function, which may be computed numerically as the gradient of the gradient. Unfortunately, this computation is generally a formidable numerical problem and, as it is required at each iteration, makes the algorithm slow and possibly unreliable. However, when it works smoothly, the Newton algorithm may converge in fewer iterations than other methods.

The large computational problems associated with the calculation of the Hessian in the Newton method are avoided by certain so-called quasi-Newton algorithms. These start with an initial estimate of the Hessian and employ updates that add information at each iteration without requiring the calculation of second derivatives. Although they generally need more iterations to achieve convergence than the Newton method, their numerical efficiency means that they are usually faster and, furthermore, tend to be more robust to the condition of the model and data. The OPTMUM procedure contains three such algorithms: the BFGS method due to Broyden (1967), Fletcher (1970), Goldfarb (1970) and Shanno (1970), the DFP method of Davidon (1968) and Fletcher and Powell (1963), and BFGS-SC, which is a modified BFGS algorithm in which the formula for the computation of the update of the Hessian estimate has been changed to make it scale free. In all three cases, the OPTMUM implementation of the algorithm uses the Cholesky factorization of the approximation to the Hessian in (3.3), i.e., $\mathbb{H}=\mathbb{C}^{\prime} \mathbb{C}$, before solution for $\mathbf{d}$. The BFGS algorithm is the default choice in OPTMUM, while the other five are available as options.

The OPTMUM procedure in Gauss 5 also includes a number of methods for computing the step length, $s$. The default method is called STEPBT, which is described in Dennis and Schnabel (1983). It first attempts to fit a quadratic to the objective function and computes an estimate of $s$ that minimizes the quadratic. If that fails, it tries a cubic function, which is rather more versatile in cases where the objective function is not well approximated by a quadratic. 
If STEPBT fails, then BRENT is used, a technique due to Brent (1972) that evaluates the objective function at a sequence of test values for $s$, determined by extrapolation and interpolation using the inverse of the "golden ratio", namely, the constant $\frac{(\sqrt{5}-1)}{2}=0.61803$. This method is generally more efficient than STEPBT but requires significantly more function evaluations.

If, in turn, BRENT fails, then a procedure called HALF is used. Denoting the objective function by $F(\widetilde{\boldsymbol{\theta}}+s \mathbf{d})$, this method first sets $s=1$. If $F(\widetilde{\boldsymbol{\theta}}+s \mathbf{d})<F(\widetilde{\boldsymbol{\theta}})$, then $s$ is set to 1 ; if not, then $s=0.5$ and $F(\widetilde{\boldsymbol{\theta}}+s \mathbf{d})$ is tried. The attempted step length is halved each time the objective function fails to decrease. When the function does decrease, $s$ is set to its current value. This method usually requires the fewest function evaluations but is most likely to fail to find the step length that decreases the objective function.

Thus, if HALF fails, a final search for a random direction that decreases the objective function is implemented. The radius of the random search is fixed via an important global variable in OPTMUM called _oprteps, the default value of which is 0.01 . It is, however, possible to specify any positive value for _oprteps.

\subsection{Computations and results}

The computations and results in this subsection and the following section relate to Hamilton's Example 3 concerning the post-war US Phillips curve. No results for the test statistic (2.11) are given as they derive from a simple ordinary least squares regression, which is unproblematical. However, they were checked for all of the cases considered in sections 3 and 4 and, without exception, the null of linearity was rejected. Noting that an OLS regression of inflation $\left(\pi_{t}\right)$ on unemployment $\left(u_{t}\right)$, lagged inflation $\left(\pi_{t-1}\right)$, and a time trend $(t)$ reveals statistically insignificant evidence of an inflation-unemployment trade-off using annual data for the period $t=1949$ to 1997, Hamilton (2001, Section 7) investigates whether a nonlinear relation like that defined in (2.1) and (2.2) of the specific form

$$
\pi_{t}=\mu\left(u_{t}, \pi_{t-1}, t\right)+\varepsilon_{t}
$$

might be an improvement. His results appear on page 563 of his paper. However, as previously mentioned, we were originally unable to reproduce Hamilton's results using Gauss 5 and Hamilton's data: the numerical optimization associated with the maximization of (2.10) failed. 


\subsubsection{Alternative algorithms and step lengths}

Examination of Hamilton's program revealed that it employs the BFGS algorithm and relies on the default value of _oprteps. It also appeared that it had been implemented by Hamilton using an earlier version of Gauss. Indeed, we were able to reproduce his results using Gauss 3, for example, without any modification of the program, although where he reports a value for $\widetilde{g}_{2}$ of 0.16 , we find a value of -0.16 , when rounded to two decimal places like all of his results. This difference in sign may be a typographical error in Hamilton's paper, but is of no consequence for the nonlinear inference, as both positive and negative values imply the identical value of $g_{i}^{2}$, and therefore of the likelihood function. However, when different algorithms were specified under Gauss 3 by suitable adjustments of the program code, the results, when they were produced using Hamilton's data file, were not always similar to those reported by Hamilton, as shown in Table 1. In this table, algorithms 1,2, 3, 4, 5 and 6 refer to the steepest descent, BFGS, BFGS-SC, DFP, Newton and PRCG methods, respectively; and the $g_{i}$ and $\alpha_{i}$ refer to the parameters in the nonlinear and the linear components of the conditional expectation function, respectively. The values of $i=1,2,3$ relate to $u_{t}, \pi_{t-1}$ and $t$, respectively, while $\alpha_{0}$ is the constant.

The results for BFGS (algorithm 2) in Table 1, obtained using the program in unmodified form, are those corresponding to Hamilton's published results and are included as a convenient reference. Apart from the one difference in sign for $\widetilde{g}_{2}$, they are identical to his. However, we find that BFGS-SC (algorithm 3) and PRCG (algorithm 6) fail for Hamilton's dataset, steepest descent (algorithm 1) produces noticeably different numerical results from Hamilton's, Newton (algorithm 5) produces very similar results except for the sign on the nonlinear parameter estimate $\widetilde{g}_{2}$, and DFP (algorithm 4) replicates the results of BFGS, the Hamilton case. Despite the big numerical differences in the results produced using algorithm 1, the high statistical significance of $\widetilde{g}_{3}$ remains and the inference concerning nonlinearity would be basically the same as that drawn by Hamilton.

Following an amount of experimentation involving modification of the program code to utilize different values of _oprteps, we eventually reproduced Hamilton's results using Gauss 5, though not all values of _oprteps proved successful and led to results. Table 2 contains the results for what was perhaps the most successful value for this parameter, namely, _oprteps $=0.00001$, while Table A1 in Appendix A also gives results for certain other_oprteps values, i.e., 0.001, 0.1 and 1.0. As can be see from Table 2, the results from Gauss 5, algorithm 2, are identical to those of Hamilton produced by the same algorithm in Gauss 3. The results 
from Gauss 5, algorithms 1 and 5 are similar in absolute terms to those given by algorithm 2 , but there are some sign changes on $\widetilde{g}_{2}$ and $\widetilde{g}_{3}$. In contrast to what was found using Gauss 3, there are surprisingly large numerical differences between the results from algorithms 4 and 6 and those from algorithm 2 when using Gauss 5. Despite these various changes across some algorithms and the two versions of Gauss, $\widetilde{g}_{3}$ remains the most statistically significant of the nonlinear parameter estimates, though $\widetilde{g}_{2}$ is marginally significant for most of the algorithms and _oprteps values. Algorithm 3 failed in all experiments due to a problem with the Cholesky decomposition (The Gauss diagnostic message produced was "Cholesky downdate failed".), and we suspect that this may be due to a program error in Gauss, which remains to be investigated. The numbers of iterations used by the alternative algorithms are, in relative terms, broadly in line with what was said about relative efficiencies in section 3 .

\subsubsection{Initial parameter estimates}

Initial estimates of parameters are often critical for convergence of numerical optimization procedures. The initial estimates for the $g_{i}$ parameters in Hamilton's program are identical to those given in his paper for use in testing for nonlinearity, namely, $g_{i}=\frac{2}{\sqrt{k s_{i}^{2}}}$, where $s_{i}^{2}$ is the variance of explanatory variable $x_{i}$; see section 2.3 above. While there is no information in Hamilton's paper concerning the initial value of $\zeta$, examination of his program revealed the start value $\zeta=0.5$. It was decided to investigate the effect of changes in the initial value of $\zeta$, using the steepest decent algorithm, which, according to Schoenberg (2001, p. 14), is the least affected of the algorithms by choice of starting values, and which was the only algorithm not to have failed in our earlier experiments.

Using _oprteps $=0.00001$ and a range of start values for $\zeta$ from 0.1 to 1.5 , inclusive, in steps of 0.1 , we found that failures occurred for the lower values of $0.1,0.2$ and 0.3 , but that all other values of $\zeta$ allowed results to be produced. In all successful cases, the number of iterations of algorithm 1 was 150, the default maximum. There was considerable variability in the final estimates of the $g_{i}$ parameters and their standard errors, although $\widetilde{g}_{3}$ was always significant. The results for these cases are given in Table 5.

\subsubsection{Algorithm switching}

In order to economize on the number of iterations and to examine whether the variability in parameter estimates noted in the previous subsection could be 
reduced, we extended the investigation of the effects of different start values to the use of an algorithm switching procedure involving two different methods, as described by Schoenberg (2001). This procedure begins with algorithm 1, which is robust to initial conditions, and a step length of unity. If the function fails to improve by the default amount of 0.01 , then a switch to algorithm 2 with step length BRENT takes place. We also explored the use of algorithm 5 (Newton) as the second of the algorithms.

Again, the procedure fails for initial values of $\zeta$ of $0.1,0.2$ and 0.3 , but produces estimates for all other start values of $\zeta$ examined. Considerably fewer iterations are involved in obtaining the final estimates, with a maximum of 29 iterations for initial $\zeta=0.6,0.7$ and 0.8 , and a minimum of 11 iterations for initial $\zeta=$ 1.1. Somewhat fewer iterations are required in all cases but one when the second algorithm is chosen to be Newton. Greatly reduced variability is observed in the final estimates and their standard errors across all cases, and these estimates are very close to Hamilton's original results as given in Table 2. Indeed, to three decimal places, the absolute values of the estimates and their standard errors are identical, save for the case of $\widetilde{\alpha}_{0}$, where the differences are none the less very small. Details of the results for the two versions of the algorithm switching experiment for the odd start values of $\zeta$ are presented in Tables 6 and 7 . Results for the even start values, which are similar to those contained in the tables, are available on request from the authors.

\section{Sensitivity to Data}

This section reports on the performance of the program and Gauss algorithms, and the results produced, when various small changes are made to the dataset used in Hamilton's Example 3. Three kinds of change were considered. The first deleted observations at the start of the dataset and the second deleted observations at the end to give successively smaller samples. The third added new observations to create successively larger, more up-to-date samples. The additional US unemployment data were obtained from http://www.bls.gov/cps/cpsdat1.pdf and the new values for the US consumer price index from ftp://ftp.bls.gov/pab/special.requests/cpi/ cpiai.txt. Checks confirmed that the observations for the period 1949 to 1997, also available from these websites, were identical to those in Hamilton's dataset. In line with Hamilton's treatment, no re-scaling was undertaken.

In all, ten alternative samples were used. Hamilton's original dataset is called dataset 1. Deleting the first observation from Hamilton's data gives dataset 2; deleting the first and second observations gives dataset 3; deleting the first, second 
and third observations gives dataset 4. Similarly, deleting the last observation gives dataset 5; deleting the last two observations gives dataset 6 ; deleting the last three observations gives dataset 7. Finally, adding the observation for 1998 gives dataset 8; adding the two observations for 1998 and 1999 gives dataset 9; adding the three observations for 1998, 1999 and 2000 gives dataset 10; and adding the four observations for 1998 to 2001, inclusive, gives dataset 11.

For each of the 10 alternative samples, Hamilton's program was implemented using Gauss 5 and the values for _oprteps of 0.00001, 0.001, 0.1 and 1.0, which were referred to in the previous section and appear in Table A1. The Gauss 3 implementation was also used with datasets 2, 5, 7, 8 and 11. A large volume of results was therefore produced and the relevant details are tabulated in Appendix B. Table 3 summarizes the nonlinear estimates given by the Gauss 3 implementation of the program using dataset 2 . These results are typical in regard to the incidence of failure of nonlinear optimization algorithms and of the differences in the results produced by different algorithms that did not fail. The results from Gauss 3 for data sets 5, 7, 8 and 11 are contained in tables B11, B12, B13 and B14, respectively, and the level of program failure in each case is at least as great as that observed using dataset 2 .

As can be seen from Table 3, algorithm 2, which is Hamilton's default method, as well as algorithms 3 and 4, fail in Gauss 3. The reason is that, after one or several iterations, the algorithm encountered a non-positive definite matrix. One or other of two Gauss diagnostic messages were obtained in this event. The first was "Negative of Hessian is not positive definite"; the second was "Matrix not positive definite". Of the methods that worked, algorithm 1 and 6 give similar results but algorithm 5 gives very different results from these, including different signs for all of the $\widetilde{g}_{i}$ coefficients. These differences are noteworthy, as is the fact that algorithm 2 fails for all of the modified datasets examined.

The details concerning the Gauss 5 implementation using datasets 2 to 11, inclusive, are contained in tables B1 to B10, respectively. For convenience, the information from these tables on success and failure of the algorithms is summarized in Table 4, along with similar information for Hamilton's data (dataset 1). Of the 264 program runs, 102 or 39 per cent failed to produce nonlinear estimates. At the extremes, algorithm 3 (BFGS-SC) failed in all cases, while algorithm 1 (steepest descent) was successful in all cases, albeit requiring the maximum number of iterations permitted. Increasing the maximum number of iterations to 250, for those algorithms that reached the original maximum of 150 , did not alter the results obtained to three places of decimals. Algorithm 5 (Newton) was the most efficient 
in terms of number of iterations but it failed in 10 out of 44 runs, i.e., in 23 per cent of cases. Algorithm 2 (BFGS), the default in Hamilton's program, failed in 28 out of 44 runs or 64 per cent of cases.

As noted in the case of Gauss 3, there are also many differences in the nonlinear estimates obtained from a given data set when different algorithms work under Gauss 5, including some sign changes. There are also some big changes in numerical estimates of parameters, including sign changes, when marginal changes in the dataset, such as the addition or deletion of just one observation, are made. The reader's attention is also drawn to the interesting results obtained using dataset 11 and algorithm 2, where the addition of four extra observations leads to radically different conclusions concerning nonlinearity than those in Hamilton's example. However, the relatively high statistical significance of $\widetilde{g}_{3}$ is generally maintained across the range of experiments we have conducted.

The algorithm switching procedures were also applied to datasets 1 to 11 , inclusive. While there were a few cases of failure, the general picture to emerge was one of increased efficiency and somewhat improved numerical stability in the final parameter estimates. Detailed results are available on request from the authors.

\section{Conclusion}

In this paper we have given an account of the new approach to nonlinear econometric modelling proposed by Hamilton (2001) and briefly described some of the methods of nonlinear optimization that may be used in the Gauss computer program provided by Hamilton for the implementation of his methodology. The performance of this program has been investigated using data relating to Hamilton's example concerning the US Phillips curve, two versions of the Gauss software and a range of alternative numerical optimization options, starting values and values for the Gauss parameter _oprteps. The performance of algorithm switching procedures has also been examined. Finally, the effects of changes in the sample data on the results produced by Hamilton's procedure have been explored. The focus has designedly been on the Gauss implementation of the procedure and, while several changes in the Gauss procedures have been investigated, no attempt has been made at modification of Hamilton's methodology per se.

The results we have presented suggest some clear conclusions, which we hope will be of value to those contemplating working with Hamilton's new method. First, different algorithms used for the numerical optimization have different chances of success. Hamilton's choice of the BFGS algorithm fails in over 60 
per cent of the cases examined in our study, while the less computationally efficient steepest descent method succeeds in all cases. Secondly, when different algorithms work, they may produce significantly different numerical results and different signs for parameter estimates. Thirdly, the use of procedures that employ two algorithms and a switching criterion appears to produce far more consistent estimates than any one algorithm used on its own. Algorithm switching is also less sensitive to the choice of initial parameter estimates. Fourthly, minor changes in data can have significant effects, both in terms of whether an algorithm operates or not and, in the case of it operating, the numerical results it produces. For example, it is interesting to note that if Hamilton's data had just one less observation at either end of the sample, or one more observation at the end, his version of the program would have failed to produce nonlinear estimates, not only with Gauss 3 but also with Gauss 5 and all of the values of _oprteps used in this study. Moreover, if his dataset had contained the four additional observations for 1998 to 2001 (dataset 11), while the program would have produced results, all three nonlinear parameter estimates would have been significant, in contrast to just $\widetilde{g}_{3}$ as found in his original study (dataset 1). Thus his inferences concerning the form of nonlinearity would also have been different. However, despite the sensitivity of results to choice of algorithm, initial values and data changes, the statistical significance of the nonlinear parameter estimates, hence the inference about the form of nonlinearity, generally seems to be little affected according to the findings that we have reported. Given the on-line availability of Hamilton's program and of the data used, it is a straightforward matter to replicate all of these findings.

The present paper is only a beginning of the work advocated by Hamilton as required to establish the usefulness of his new methodology, and our empirical investigations are continuing. Indeed, the approach used above has already been extended to the implementation of Hamilton's program for his Example 1 and Example 2 [Hamilton (2001, Section 7)]. However, the findings are not discussed here, except to say the indications are that for the simulated data sets used in Examples 1 and 2, the sensitivity of results to choice of algorithm and size of sample is less pronounced than has been found using the real data of Example 3. For the interested reader, some of the results obtained using single algorithms for Examples 1 and 2 are presented in the tables in Appendix C. Further results using alternative start values and the algorithm switching procedures are available from the authors.

It is difficult to say what, precisely, is the cause of the numerical instabil- 
ity of the algorithms revealed in this study. However, useful insights into two possibilities are provided by Warnes and Ripley (1987) and Mardia and Watkins (1989). For instance, the likelihood function may be multimodal and the global maximum may not correspond to sensible values of the parameters, or the likelihood function may have a very flat ridge with a number of local maxima [Warnes and Ripley (1987, p. 640 and p. 641)]. Another possibility is that difficulties exist within Hamilton's methodology. For example, as suggested by a referee, the use of alternative covariance functions for specifying the random field regression model may help to reduce numerical instability [see Dahl and Gonzalez-Rivera (2003)], as may different procedures for estimating the parameters of Hamilton's random field regression model, such as the recently proposed two step method of Dahl and Qin (2004), which promises unique identification and good convergence properties. These matters constitute an interesting agenda for future research.

With regard to the implementation of the original Hamilton random field regression methodology, which this paper addresses, our main recommendation is to employ an algorithm switching approach supplemented as necessary by changes of the Gauss _oprteps parameter and the starting value of $\zeta$. On the basis of the evidence provided in this paper such an approach is more efficient than the use of single algorithms and appears to be less susceptible to failure. 


\section{References}

Bond, D., M.J. Harrison and E.J. O'Brien (2003): "Investigating Nonlinearity: A Note on the Implementation of Hamilton's Methodology", University of Dublin, Trinity Economic Paper No. 12.

Brent, R.P. (1972): "A New Algorithm for Minimizing a Function of Several Variables without Calculating Derivatives"; in Anderssen, R.S., L.S. Jennings and D.M. Ryan. Optimization, Brisbane: University of Queensland Press.

Brent, R.P. (1973): Algorithms for Minimization without Derivatives, Englewood Cliffs, N.J.: Prentice-Hall.

Broyden, C.G. (1967): "Quasi-Newton Methods and their Application to Function Minimization", Mathematics of Computation, 21, 368-381.

Dahl, C.M. (1998): "An Investigation of Tests for Linearity and the Accuracy of Flexible Nonlinear Inference", University of Aarhus, Working Paper.

Dahl, C.M. and G. Gonzalez-Rivera (2003): "Testing for Neglected Nonlinearity in Regression Models based on the Theory of Random Fields", Journal of Econometrics, 114, 141-164.

Dahl, C.M. and Y. Qin (2004): "The Limiting Behavior of the Estimated Parameters in a Misspecified Random Field Regression Model", Purdue University, Working Paper.

Davidon, W.C. (1968): "Variance Algorithms for Minimization", The Computer Journal, 10, 406-410.

Dennis, Jr., J.E. and S.B. Schnabel (1983): Numerical Methods for Unconstrained Optimization and Nonlinear Equations, Englewood Cliffs, NJ: PrenticeHall.

Fletcher, R. (1970): "A New Approach to Variable Metric Algorithms", The Computer Journal, 13, 317-322.

Fletcher, R. and M.J.D. Powell (1963): "A Rapidly Convergent Descent Method for Minimization", The Computer Journal, 6, 163-168.

Goldfarb, D. (1970): "A Family of Variable-Metric Methods Derived by Variational Means", Mathematics of Computation, 24, 23-26.

Greene, W.H. (2003): Econometric Analysis (5 ${ }^{\text {th }}$ edn.), Upper Saddle River, N.J.: Pearson Education, Inc.

Hamilton, J.D. (2001): "A Parametric Approach to Flexible Nonlinear Inference", Econometrica, 69, 537-573.

Mardia, K.V. and A.J. Watkins (1989): "On Multimodality of the Likelihood in the Spatial Linear Model", Biometrika, 76, 289-295. 
Murray, W. (Ed.) (1972): Numerical Methods for Unconstrained Optimization, London: Academic Press.

Polak, E. and G. Ribiere (1969): "Note Sur la Convergence de Method des Direction Conjugees", University of California, Berkeley, Department of Electrical Engineering and Computer Sciences, Working Paper.

Schoenberg, R. (2001): Optimization, Ch. 2, Gauss Reference Manual, Maple Valley, WA: Aptech Systems, Inc.

Shanno, D.F. (1970): "Conditioning of Quasi-Newton Methods for Function Minimization", Mathematics of Computation, 24, 647-656.

Warnes, J.J. and B.D. Ripley (1987): "Problems with Likelihood Estimation of Covariance Functions of Spatial Gaussian Processes", Biometrika, 74, 640-642.

Wecker, W.E. and C.F. Ansley (1983): "The Signal Extraction Approach to Nonlinear Regression and Spline Smoothing", Journal of the American Statistical Association, 78, 81-89. 


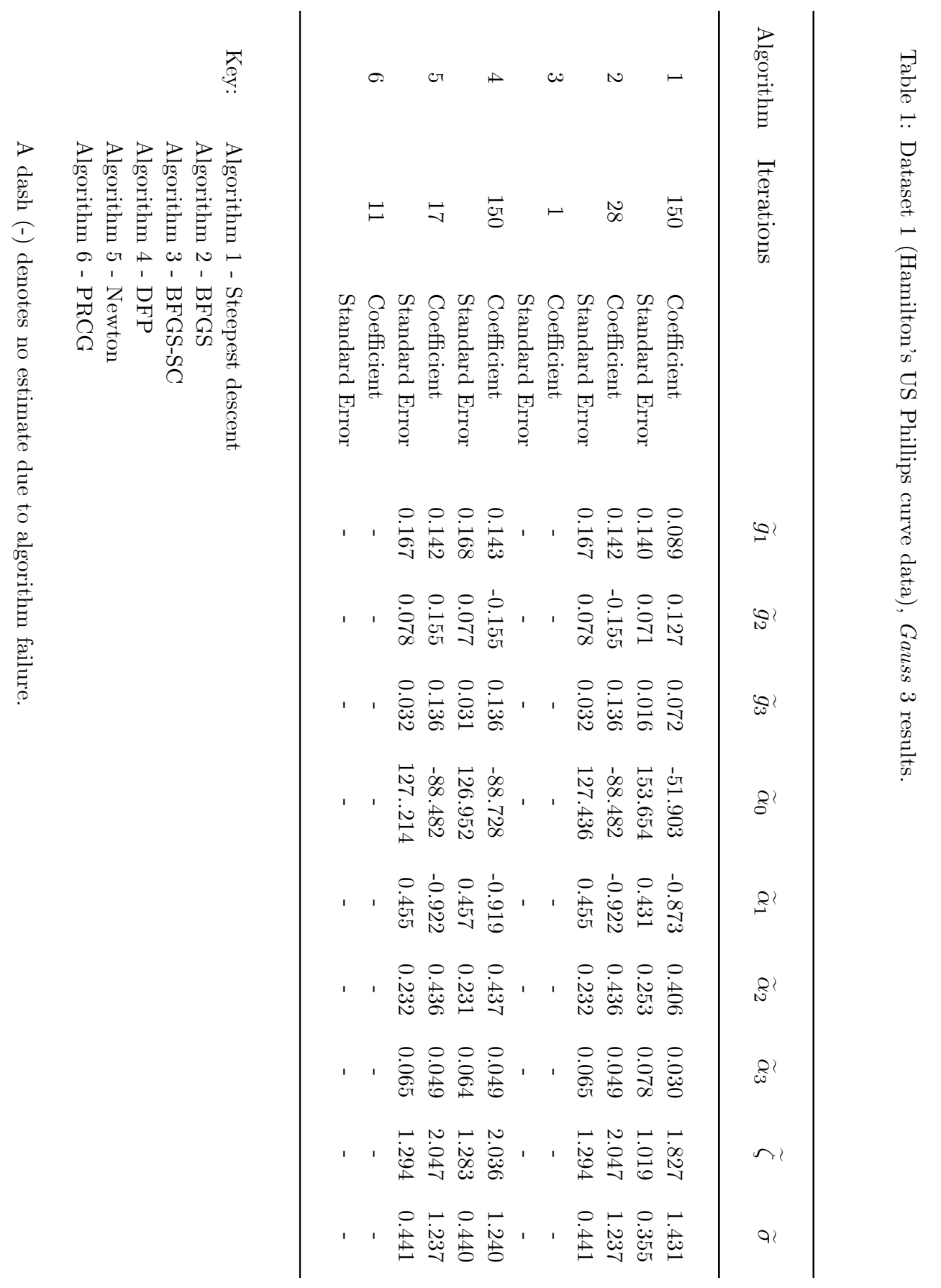




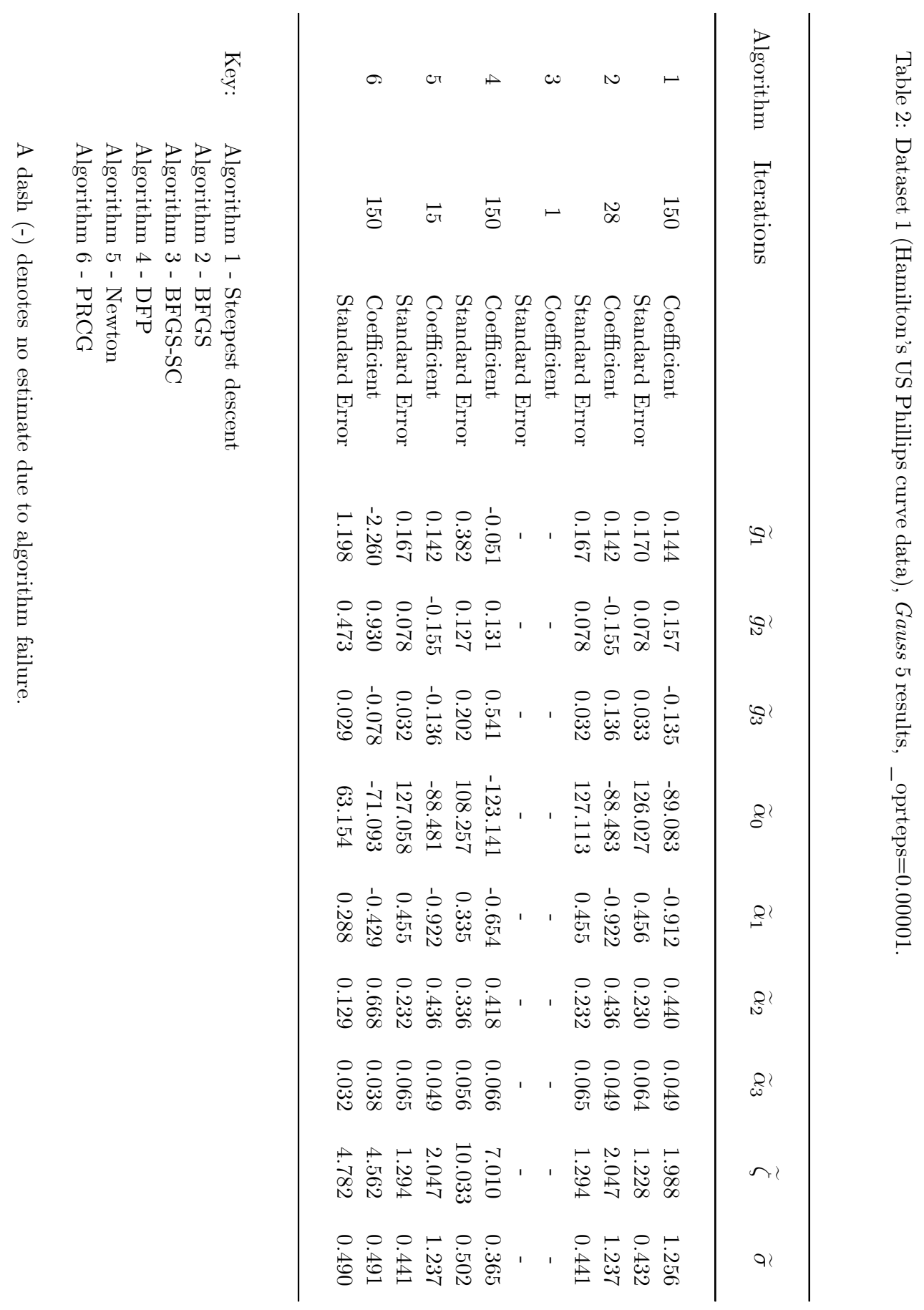




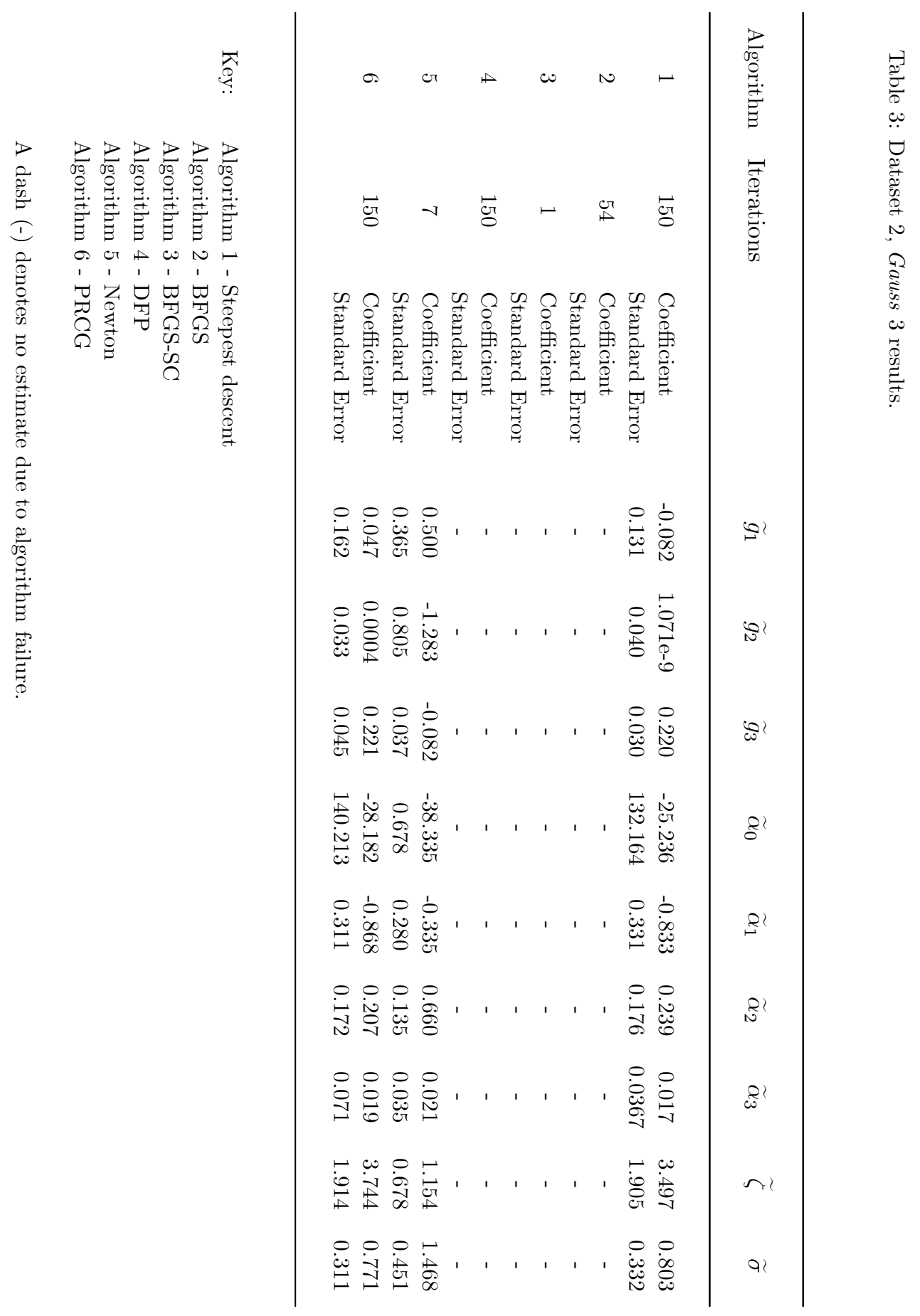




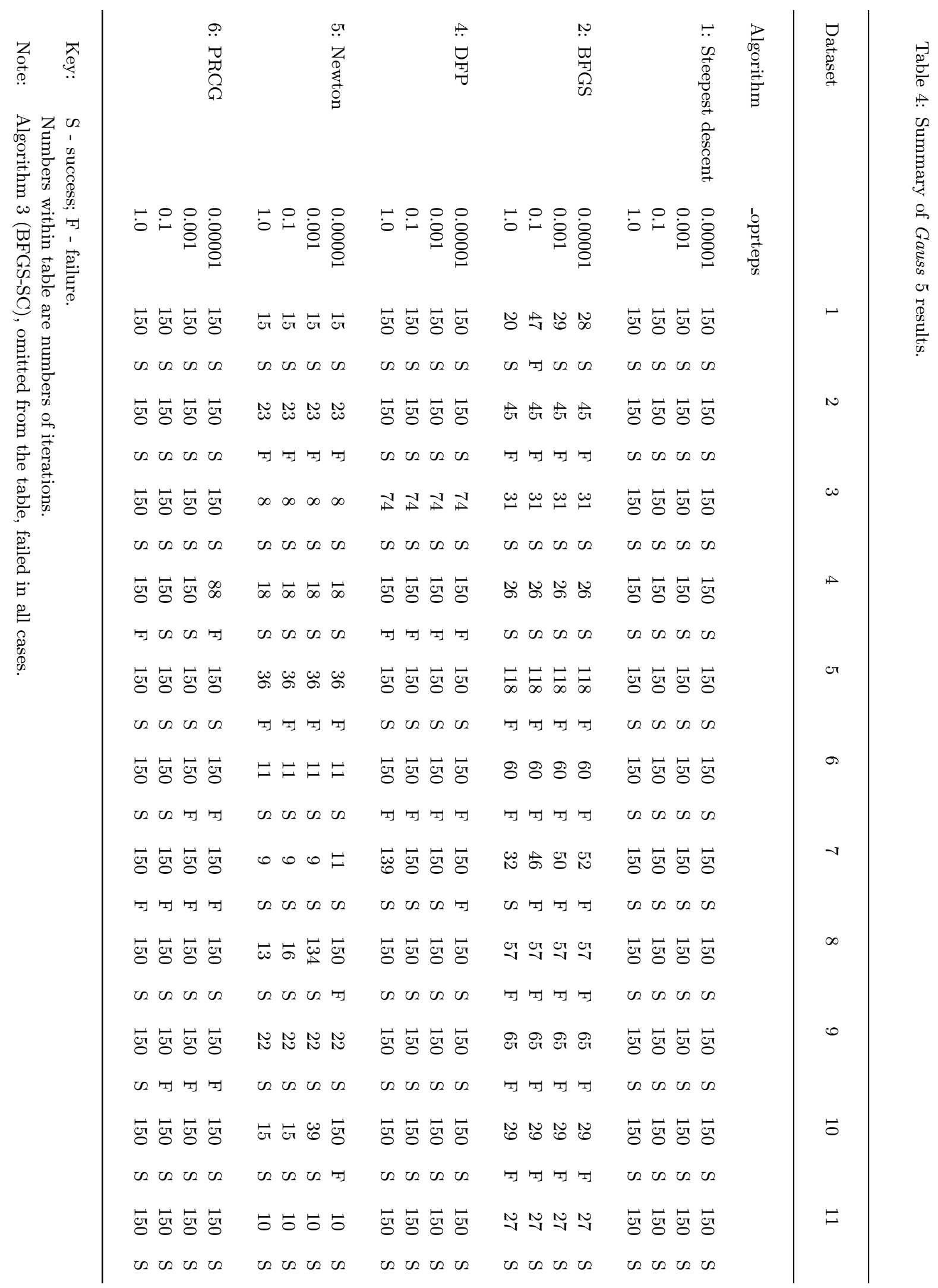




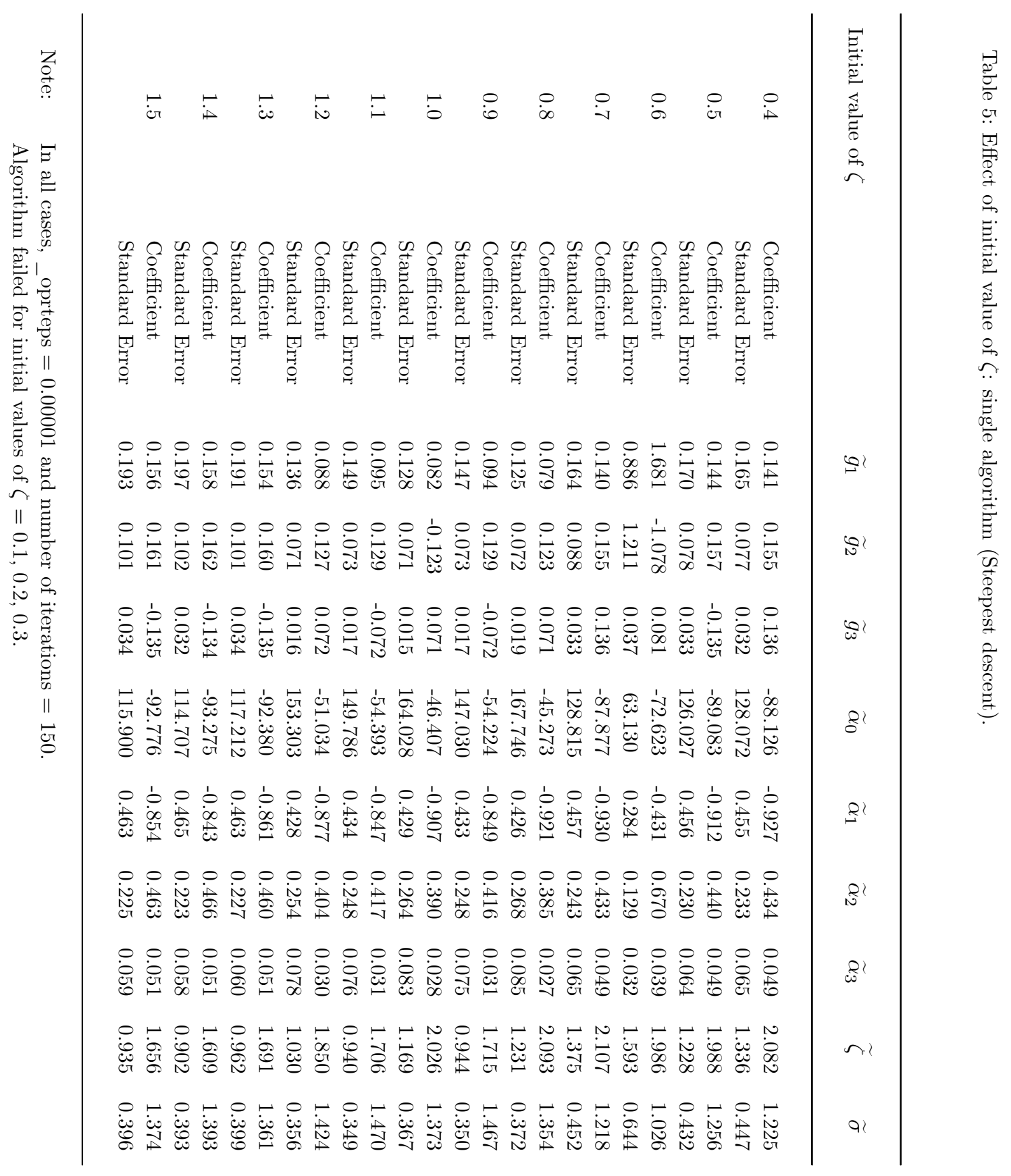




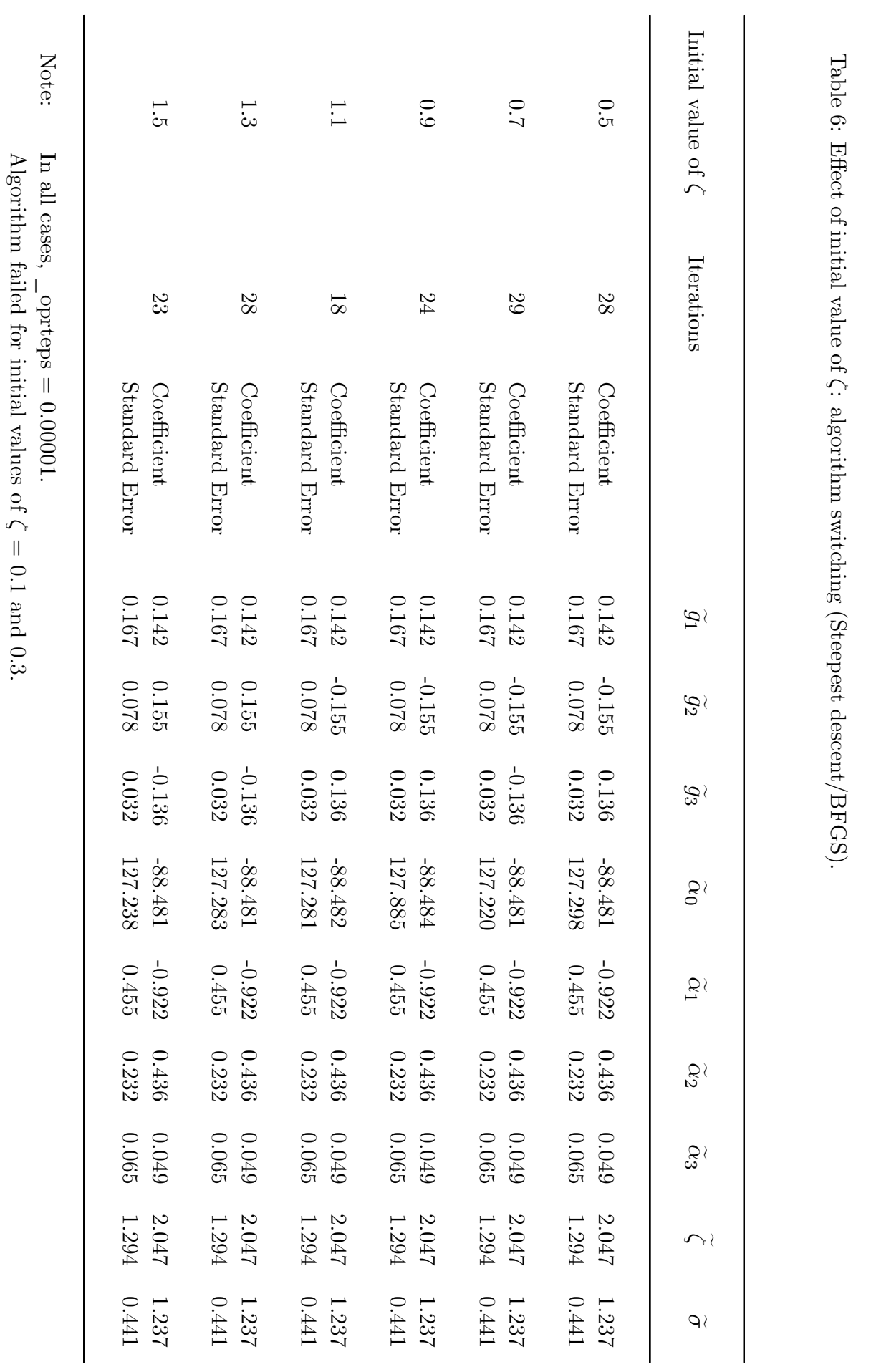




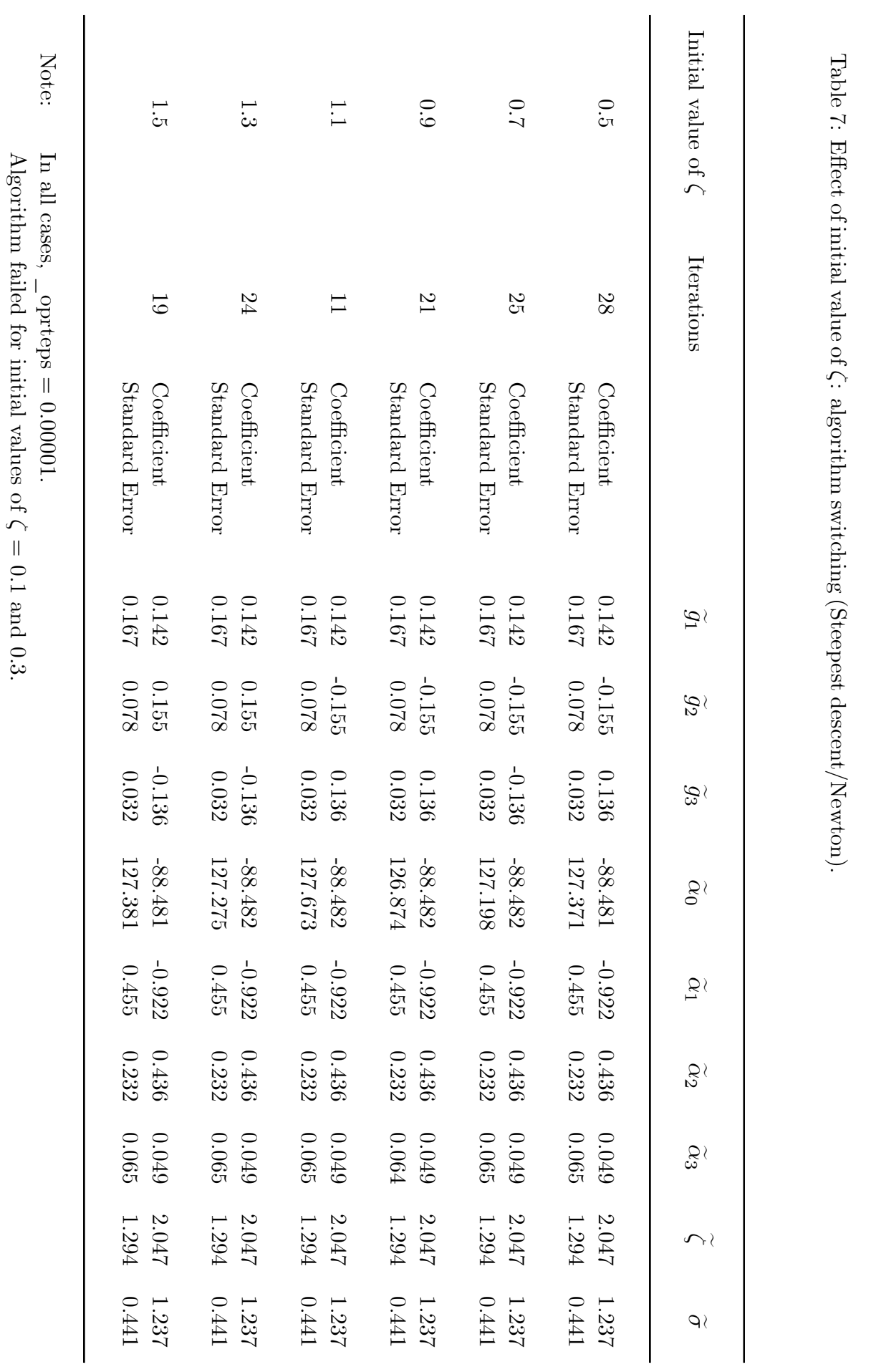


Appendix A

Summary of Gauss 5 Results. 


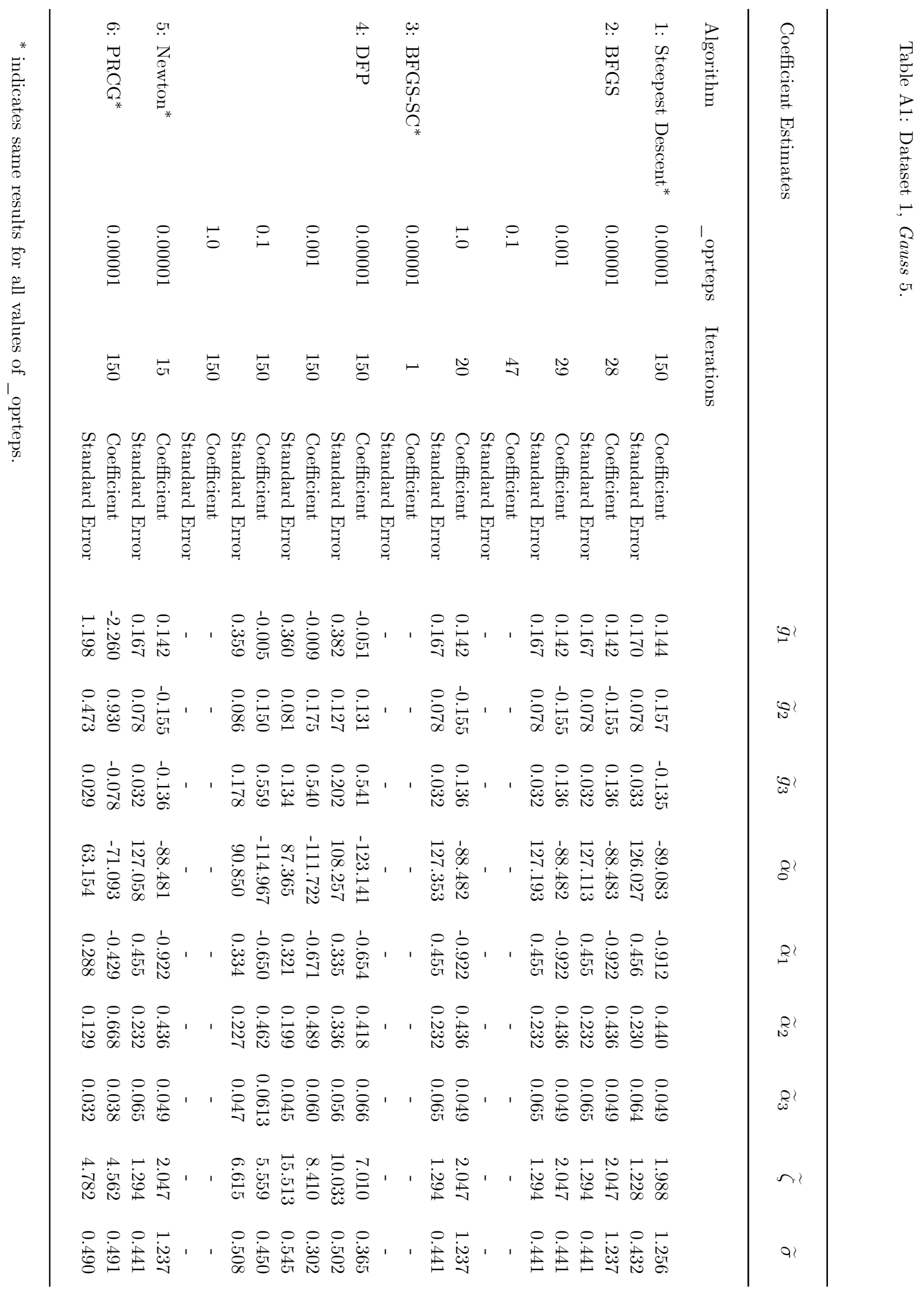


Appendix B

Detailed results, Gauss $3 \&$ 5, for all datasets. 


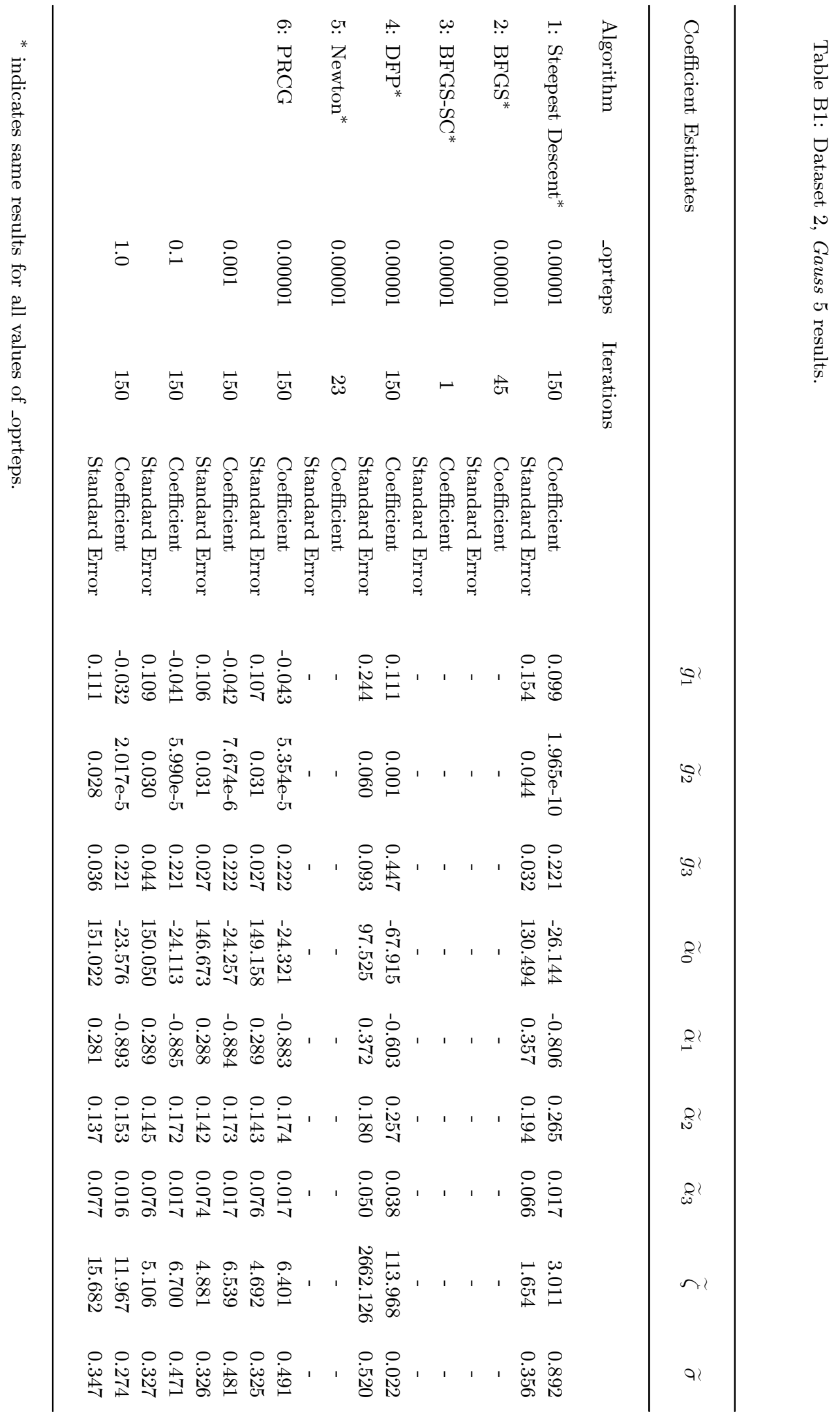




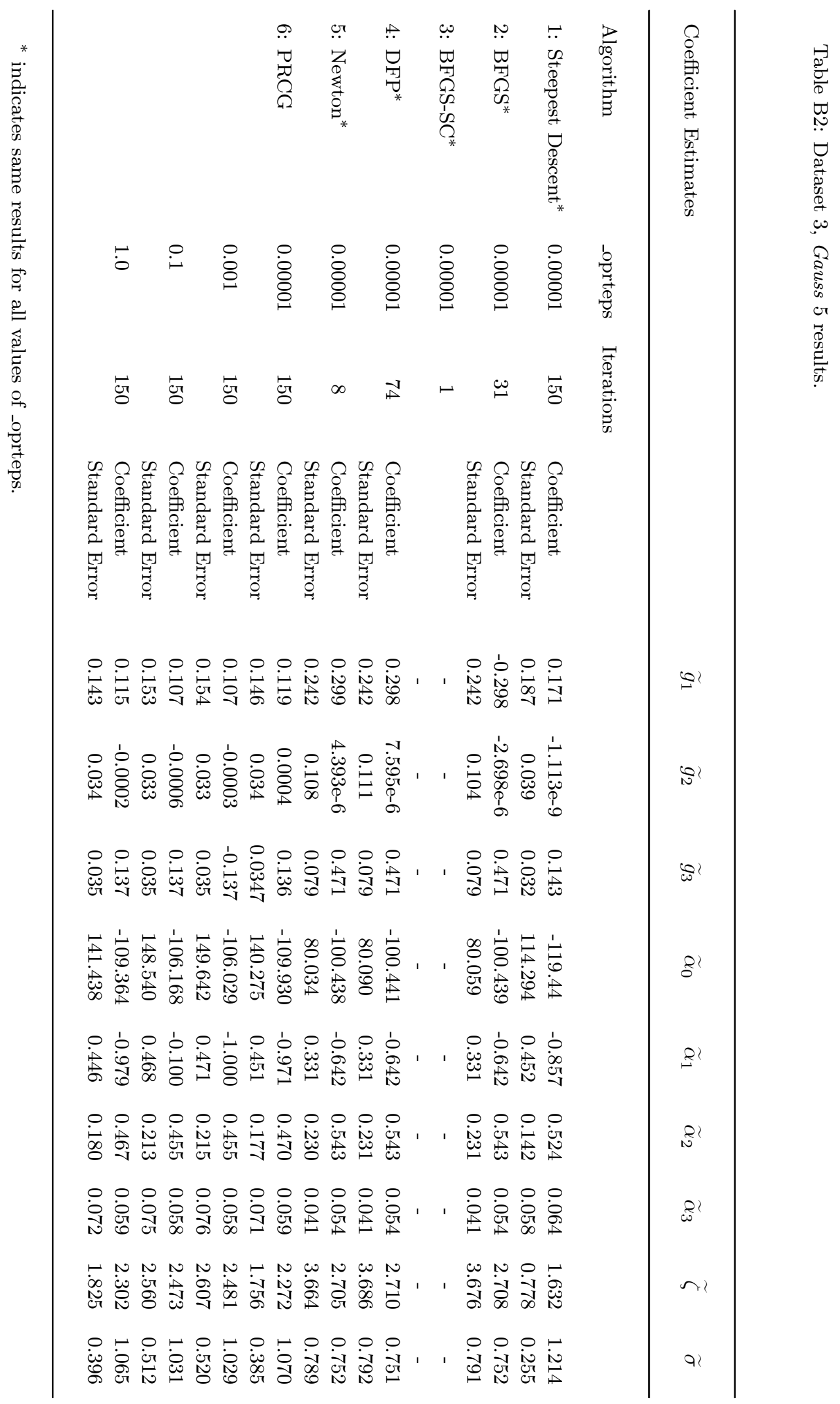




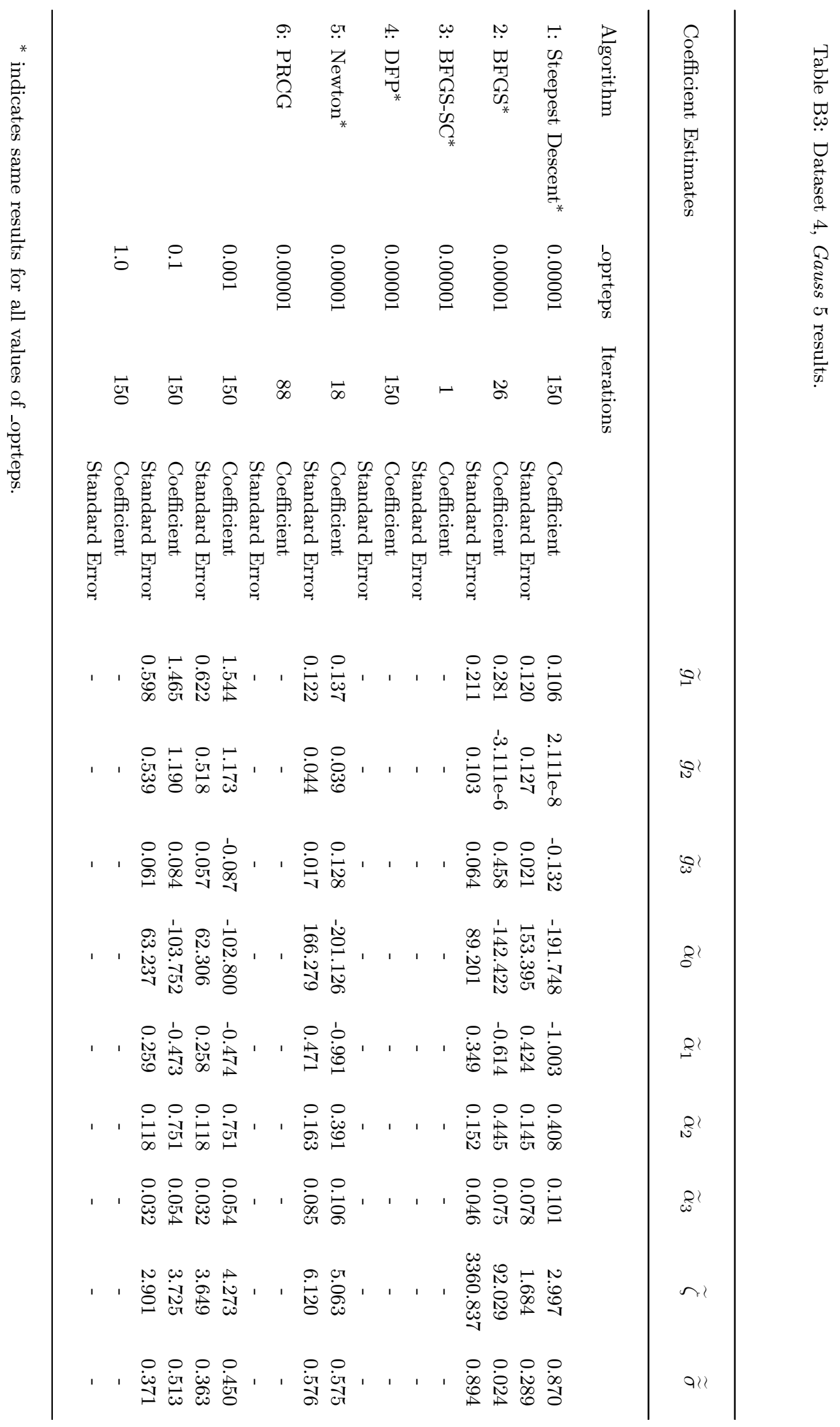




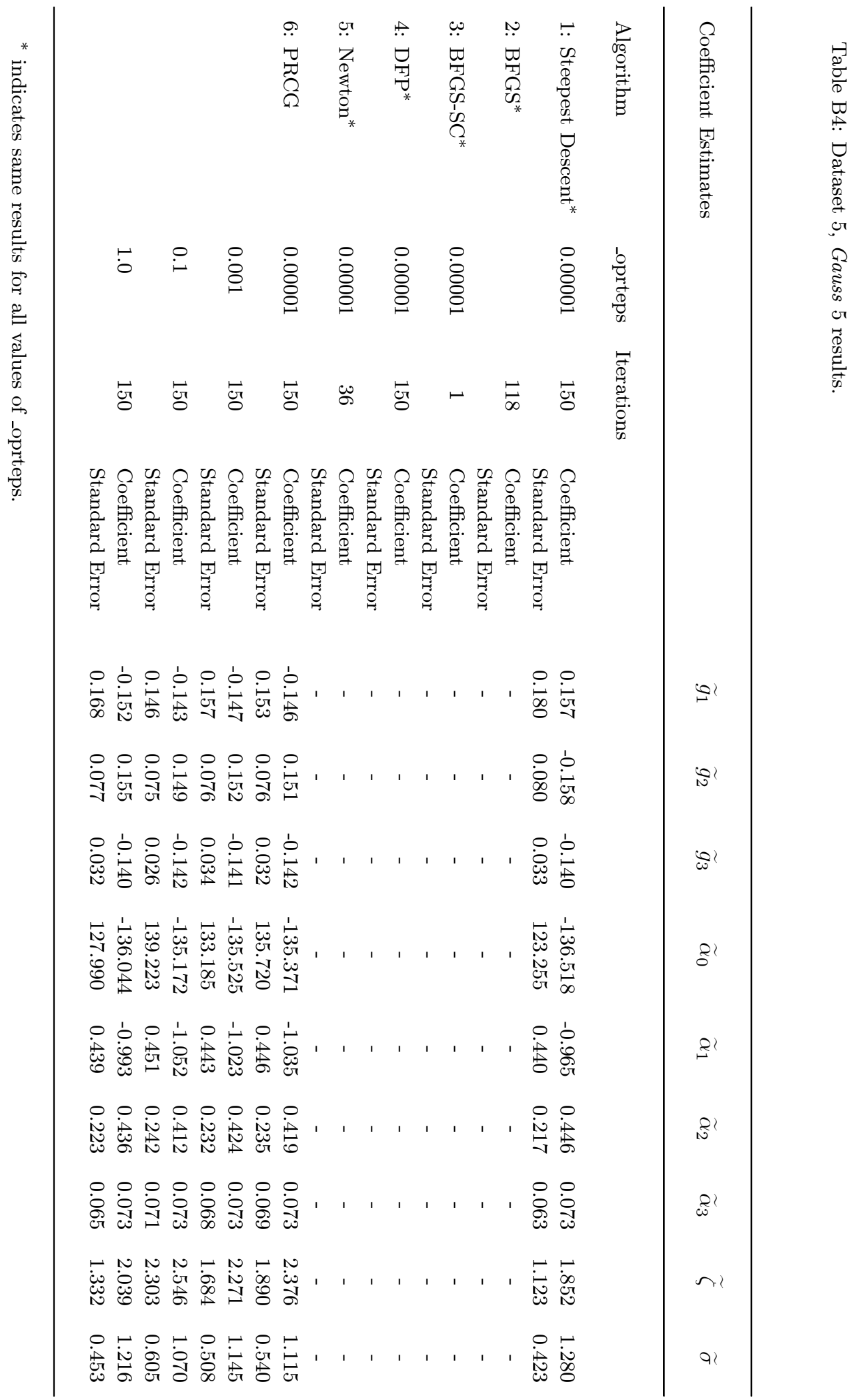




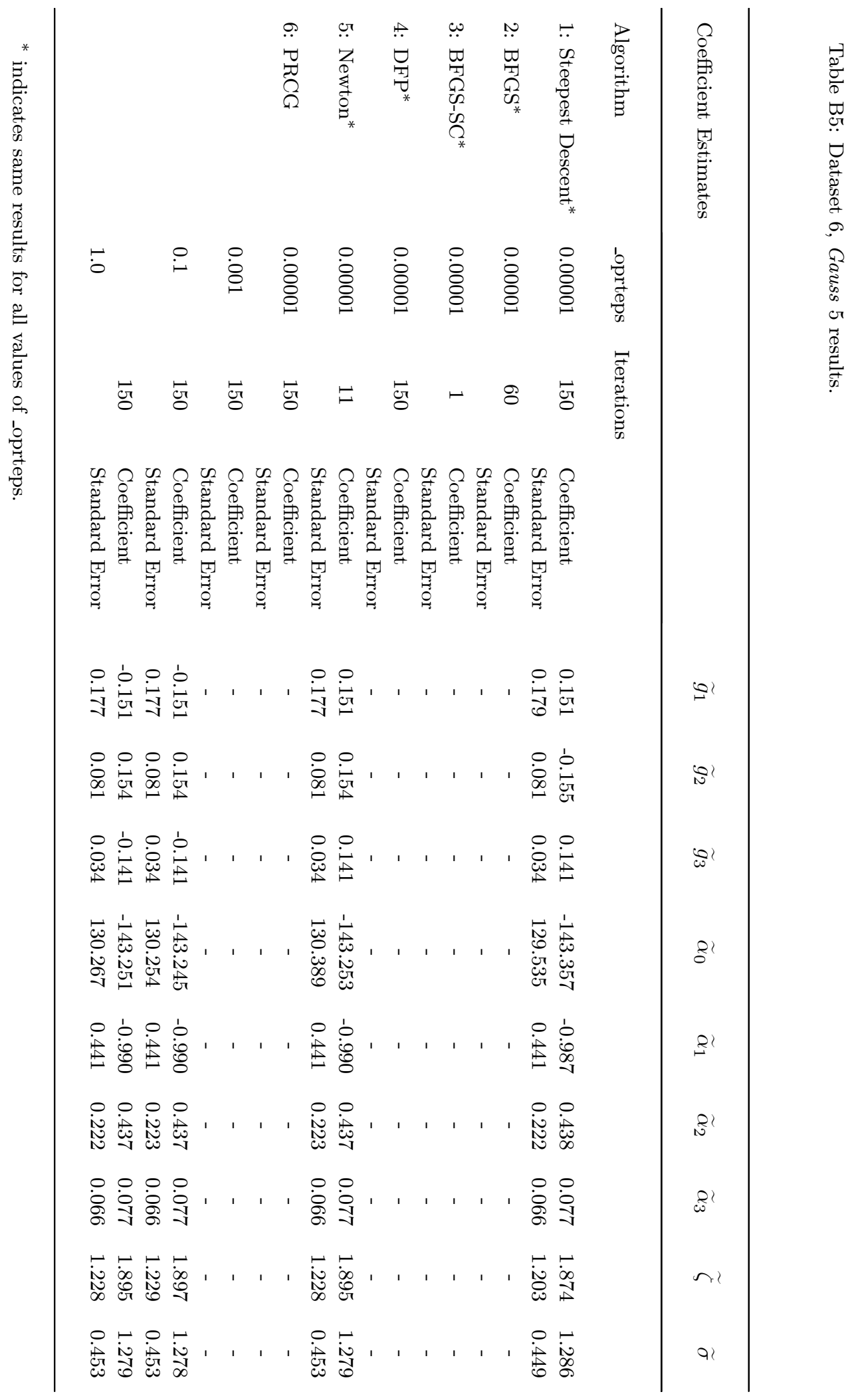




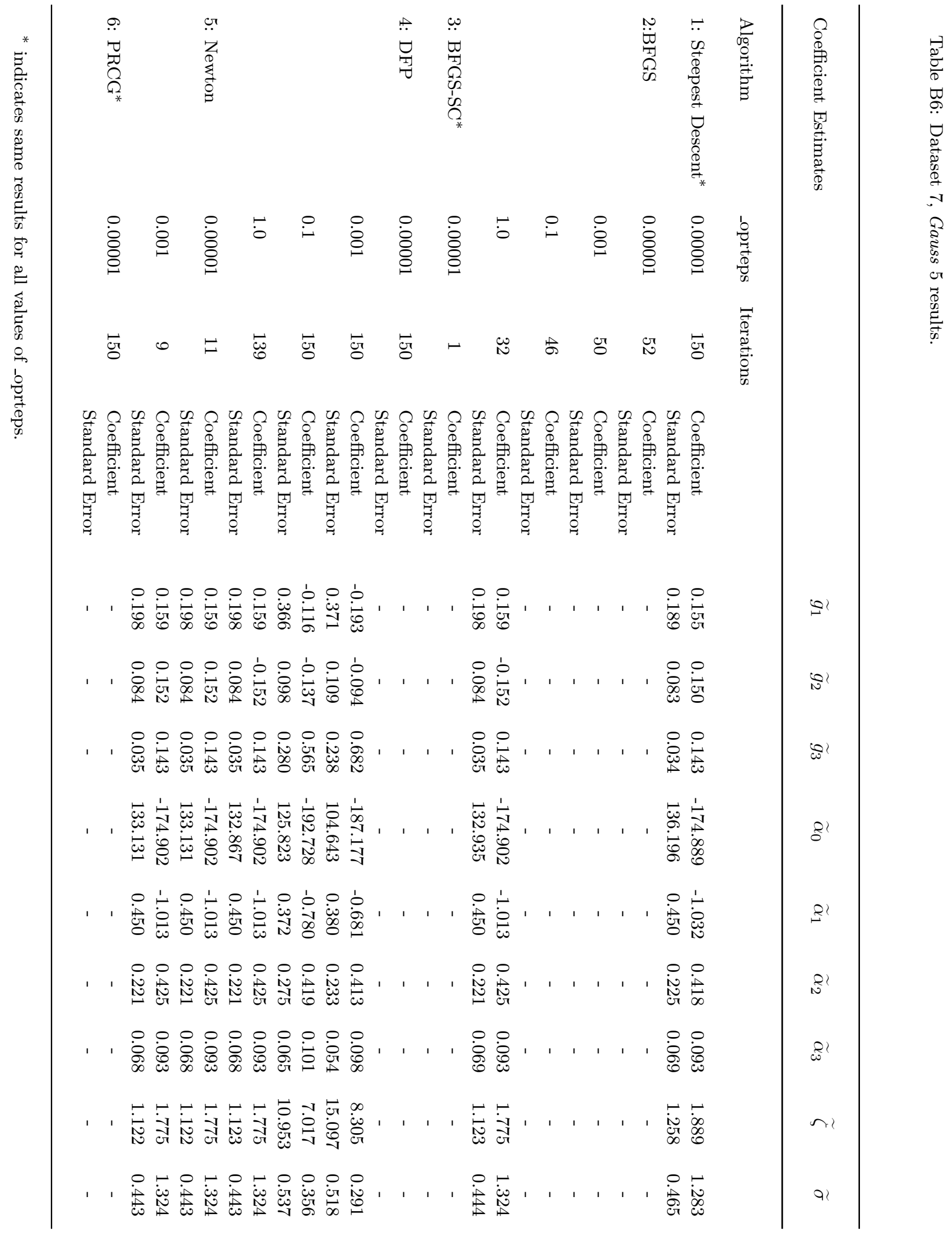




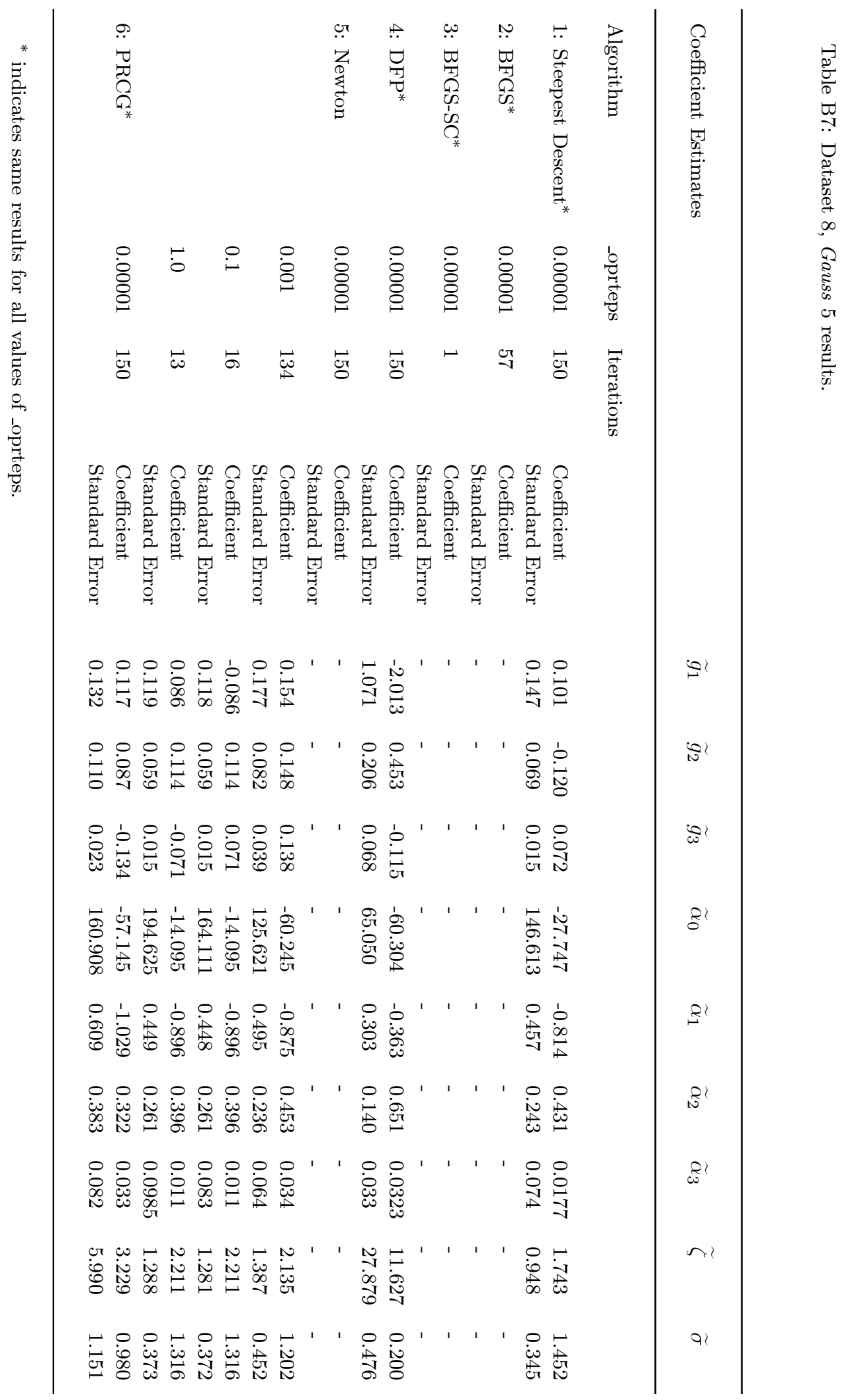




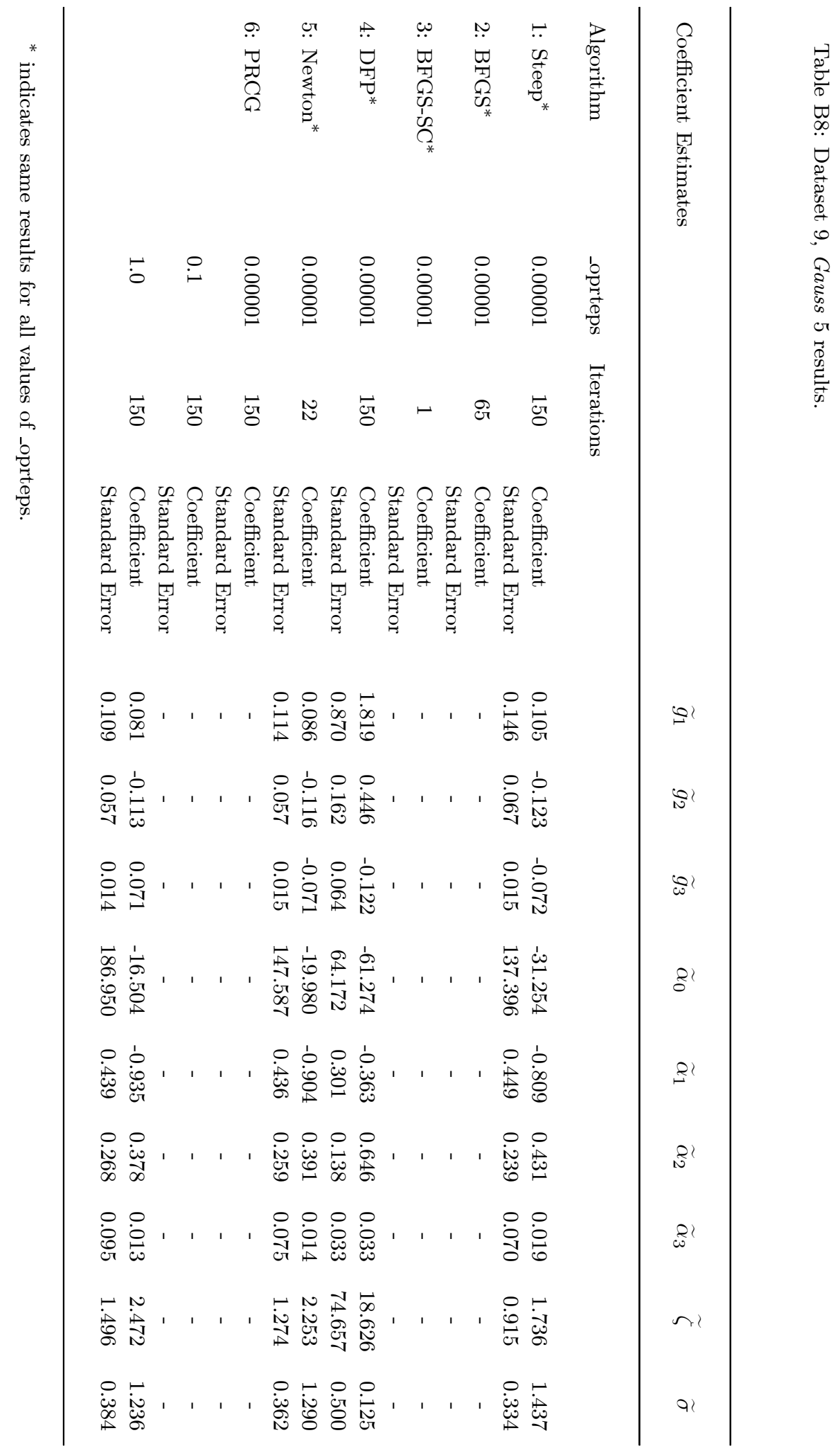




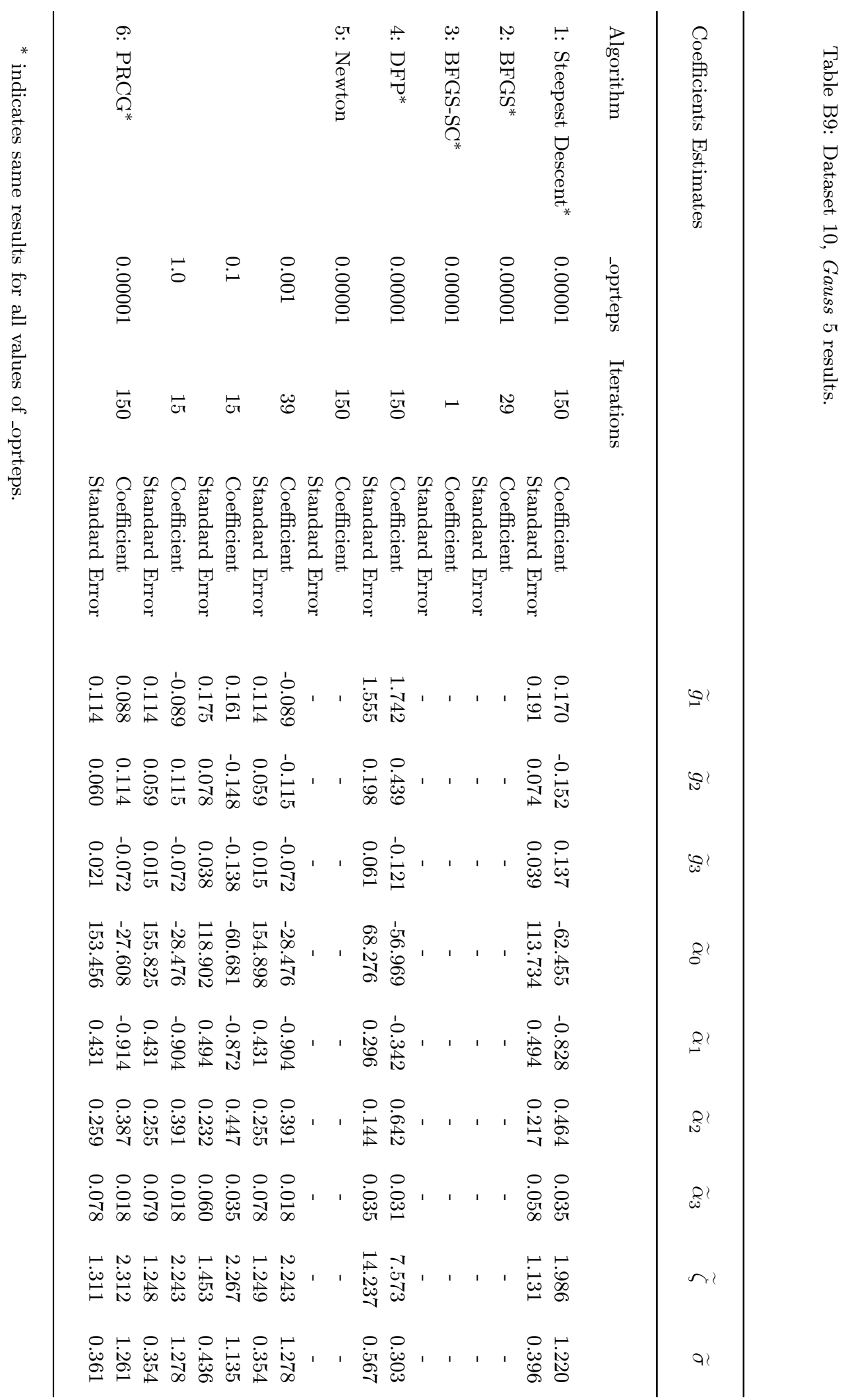




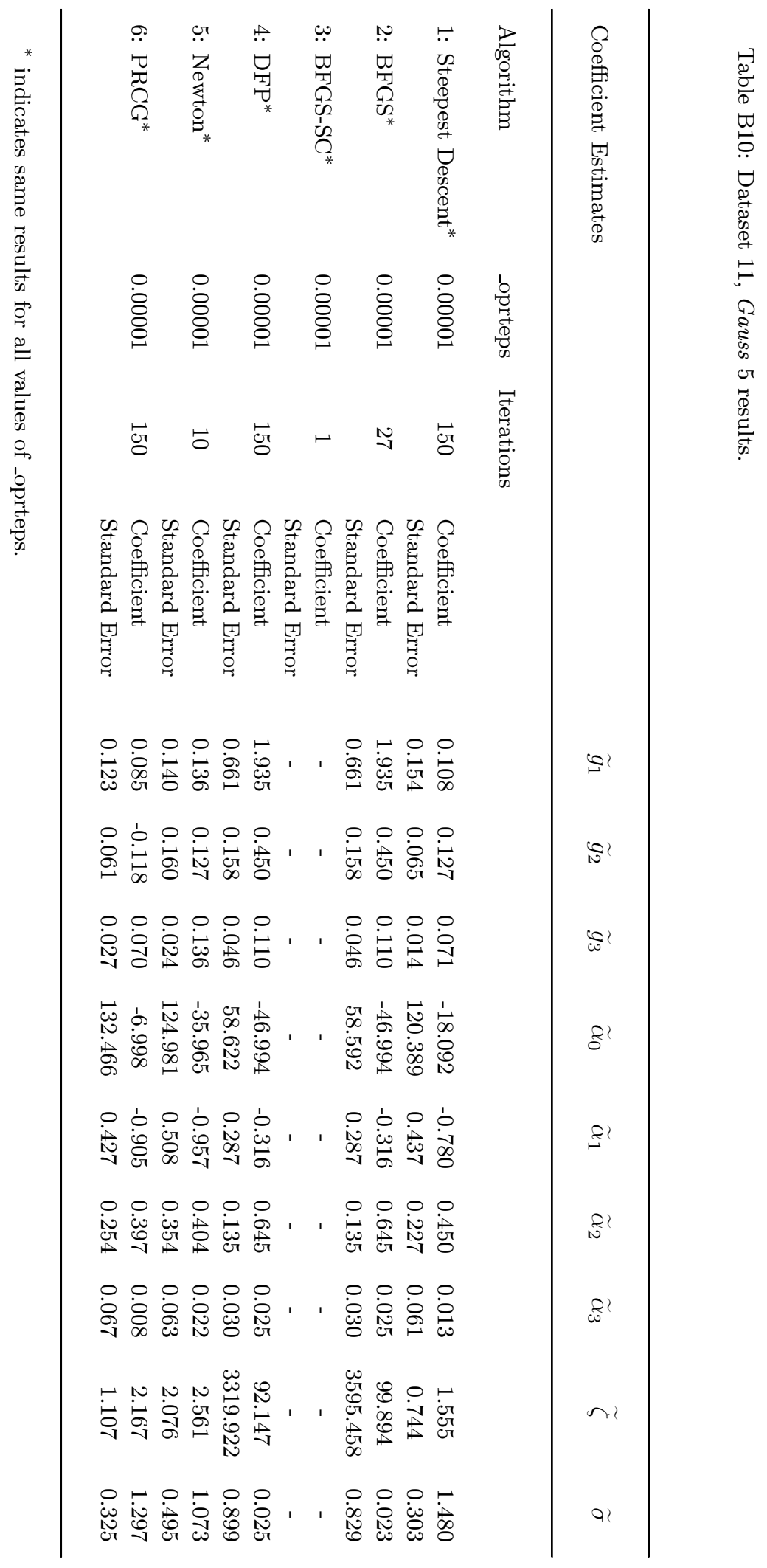




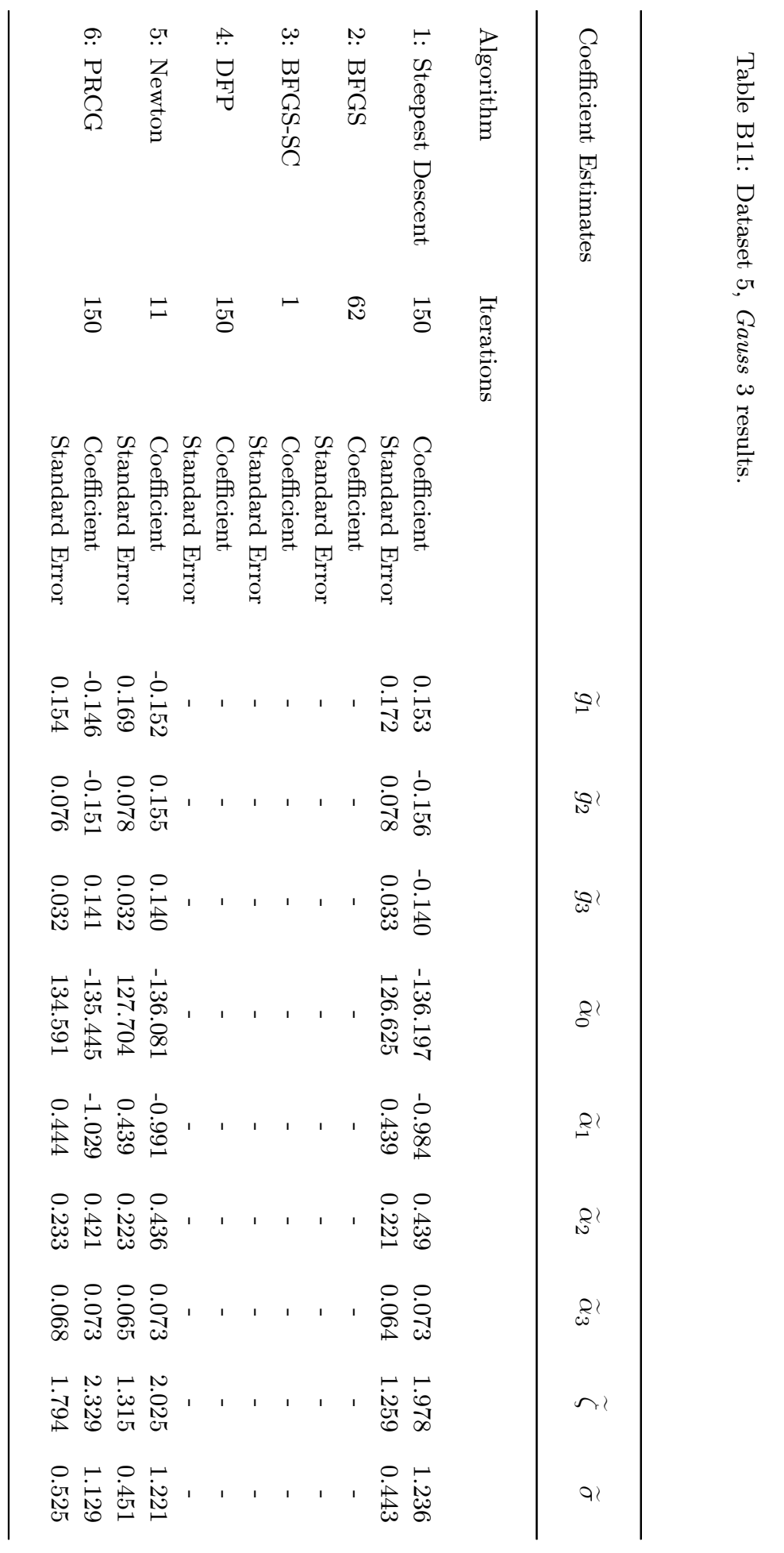




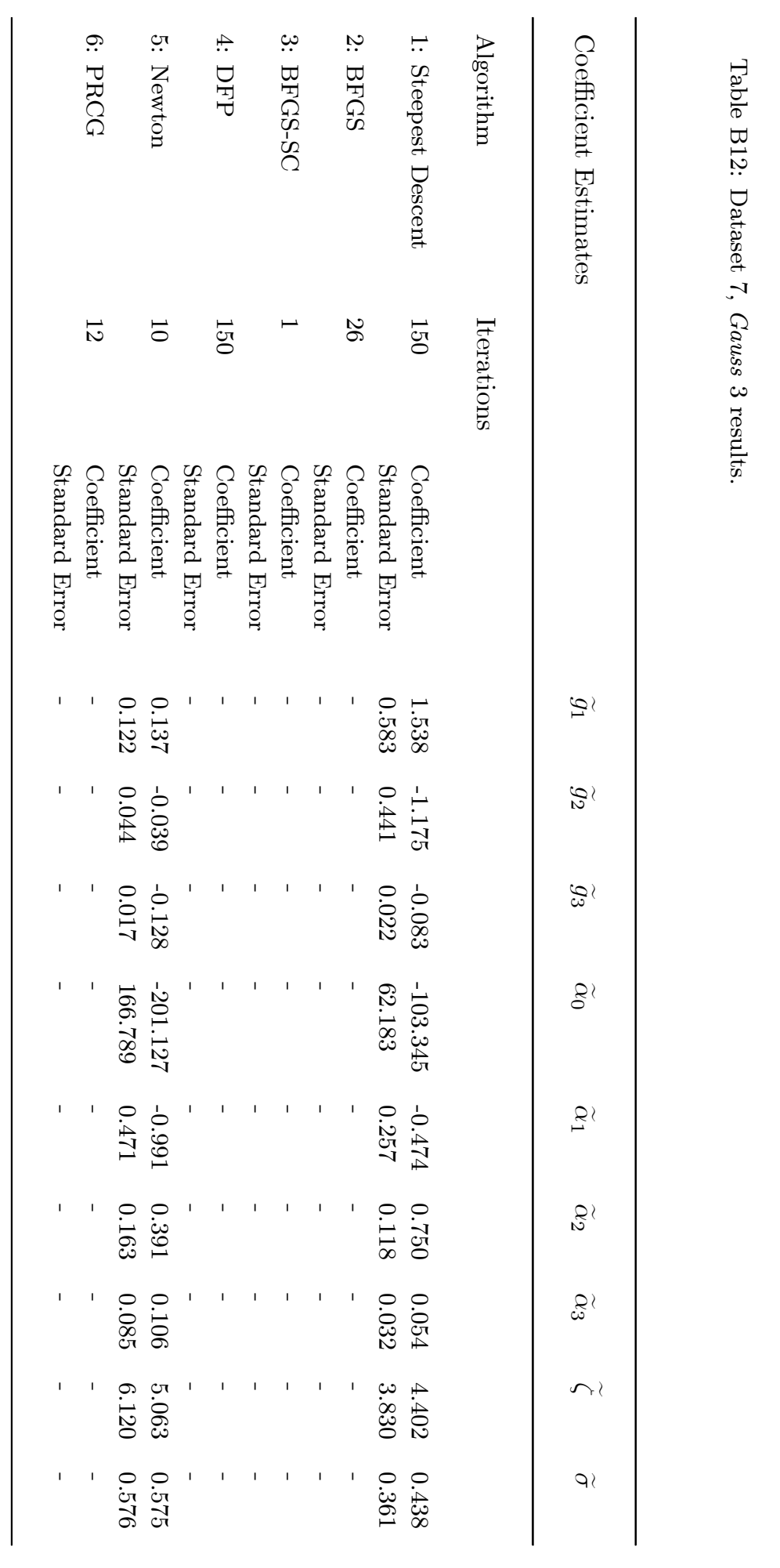




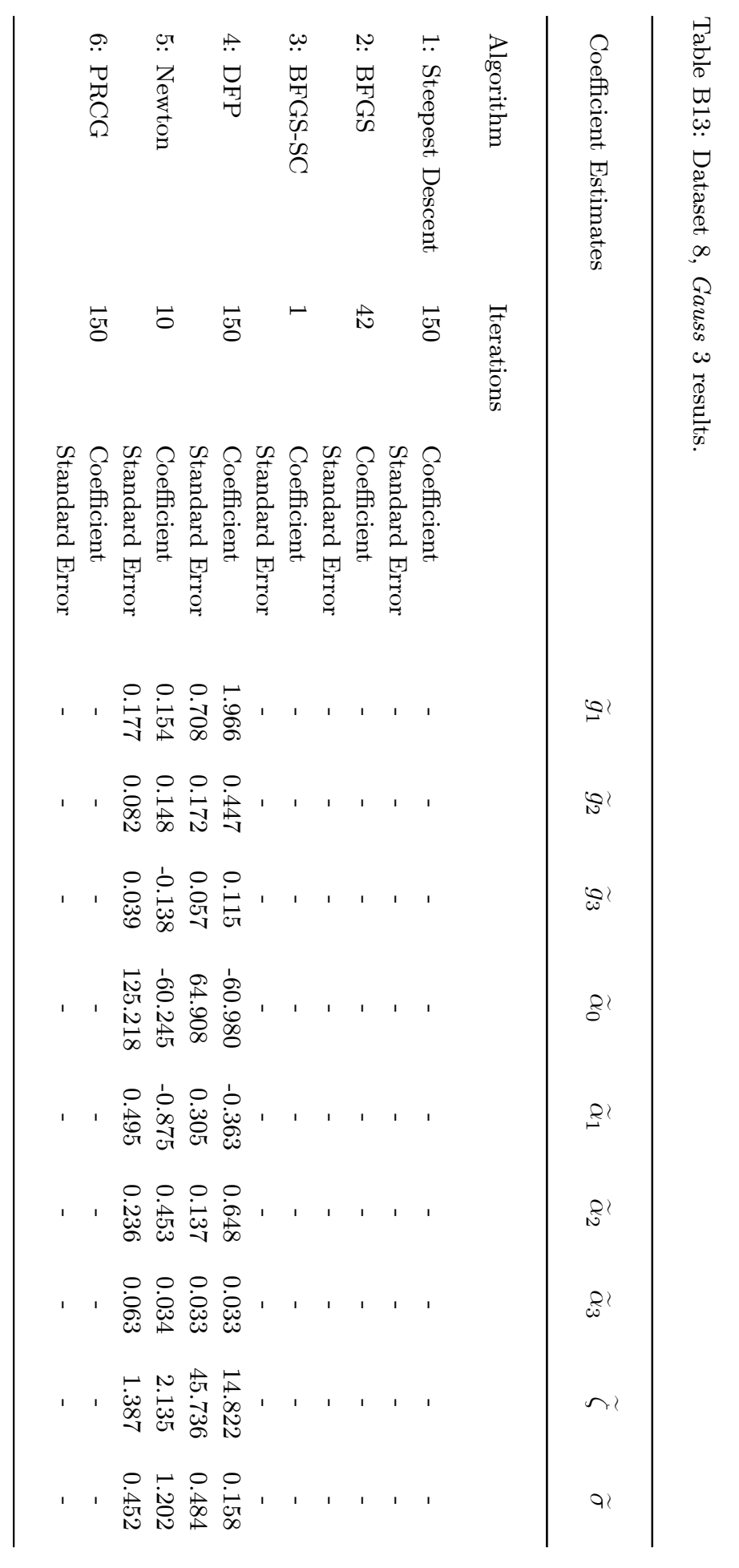




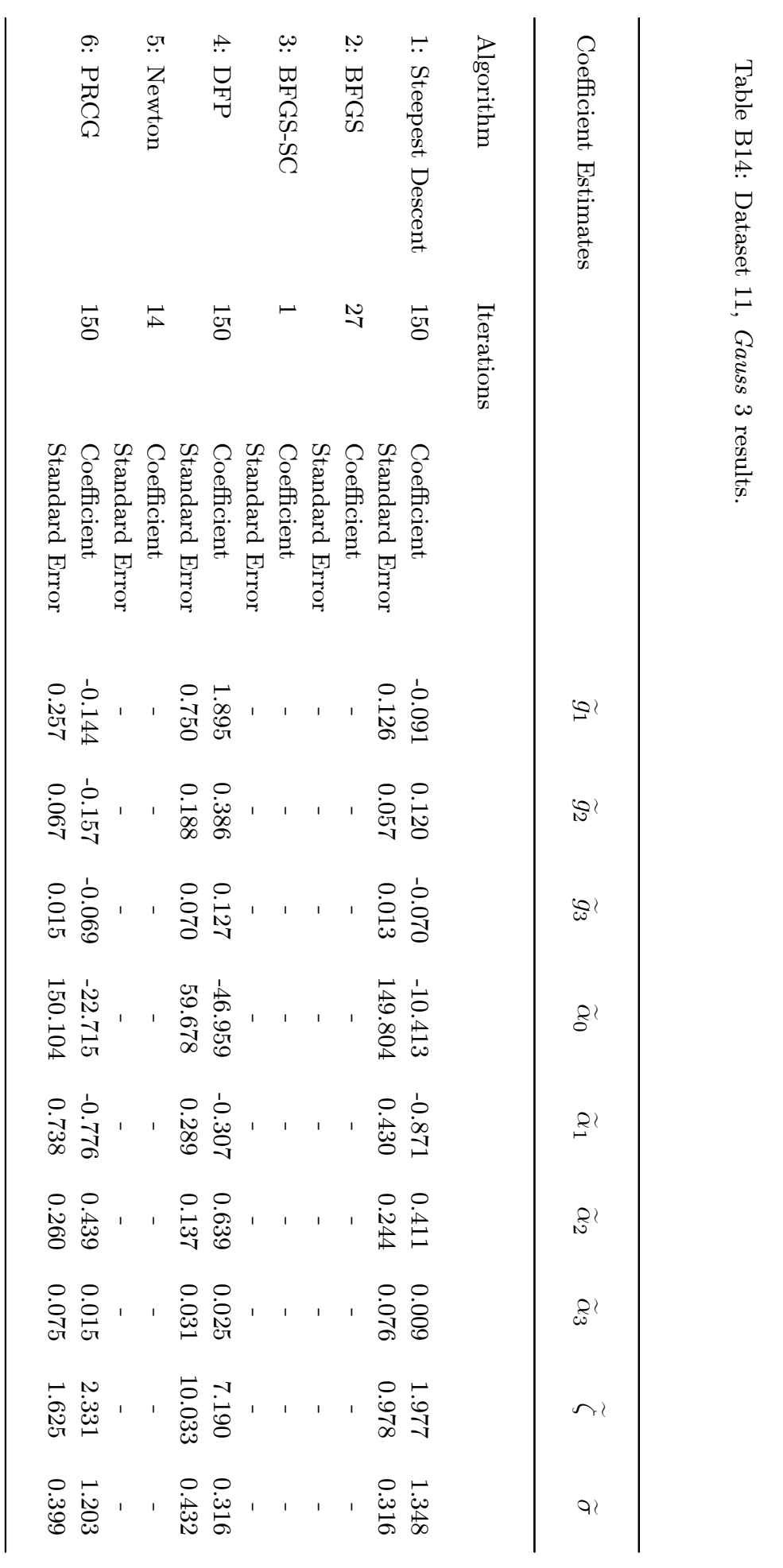




\section{Appendix C}

Hamilton's example $1 \& 2$ results. 


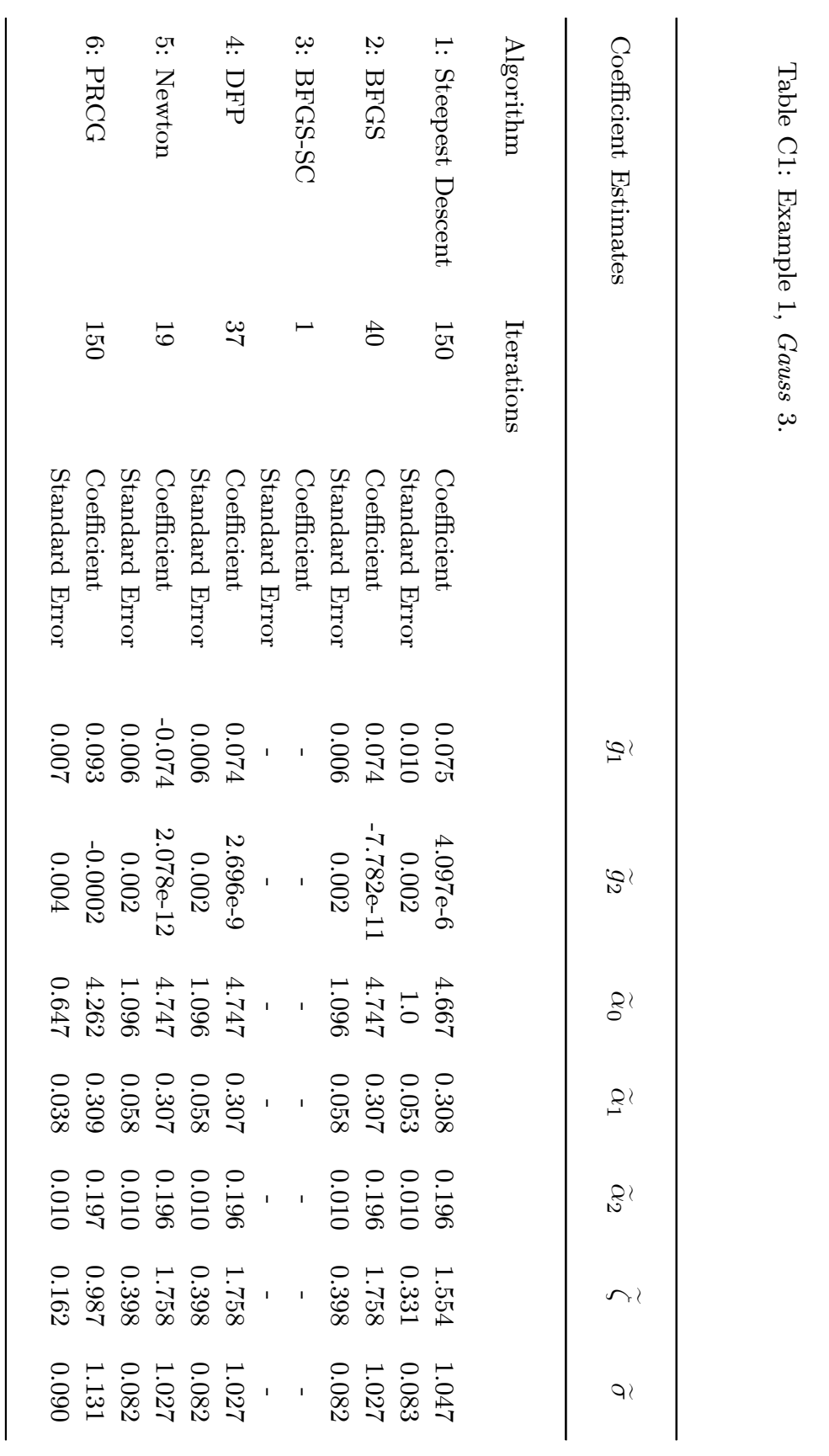




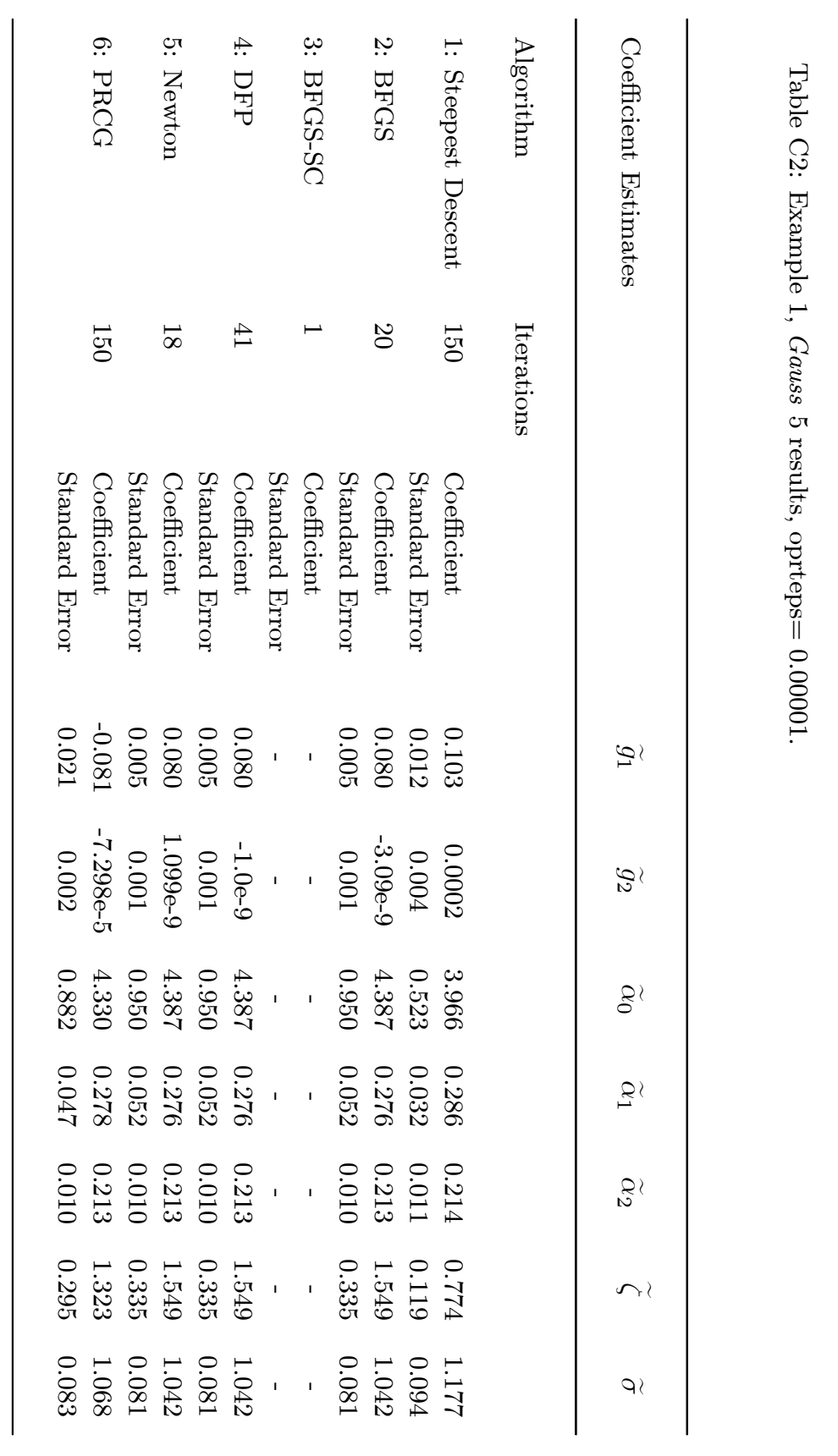




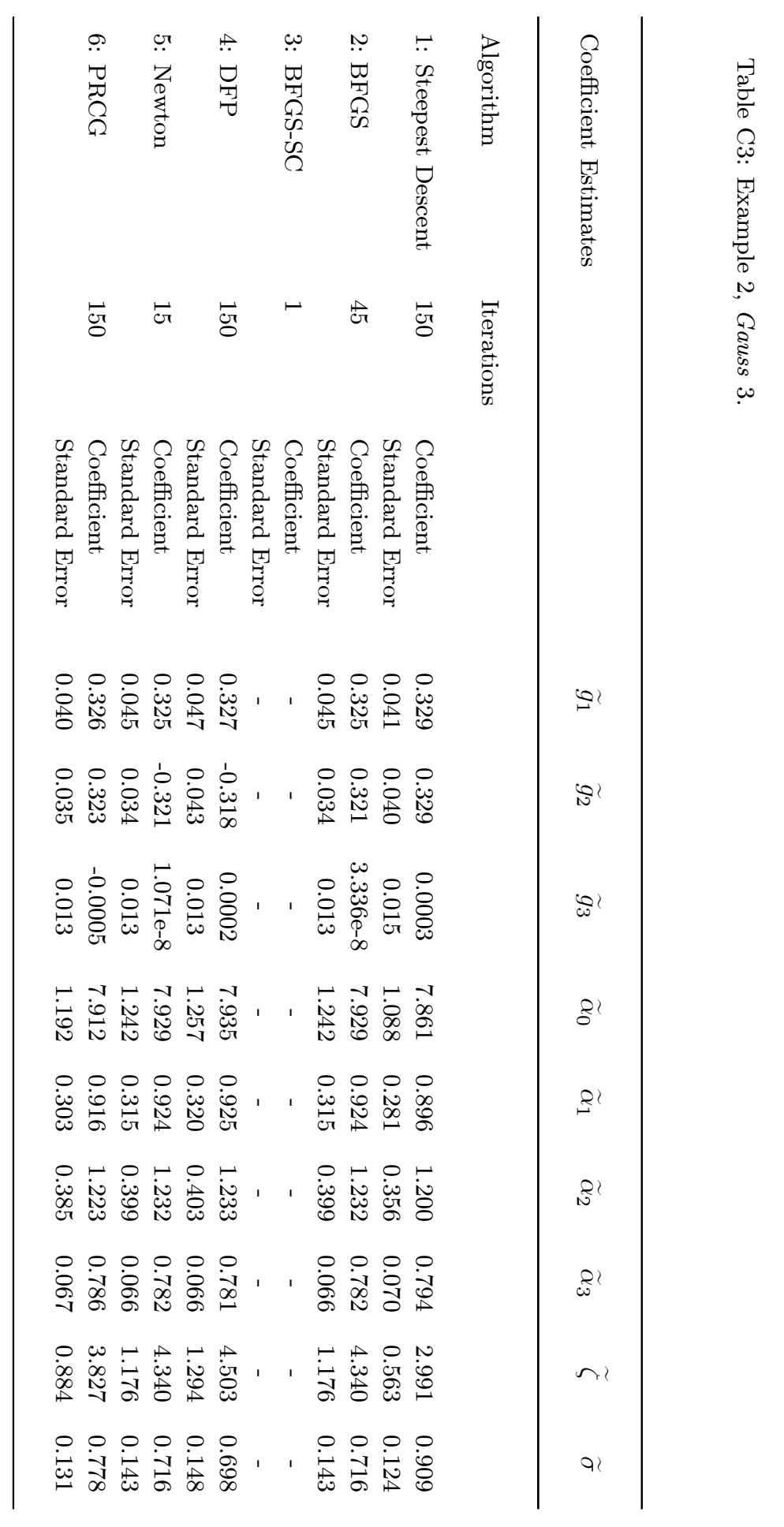




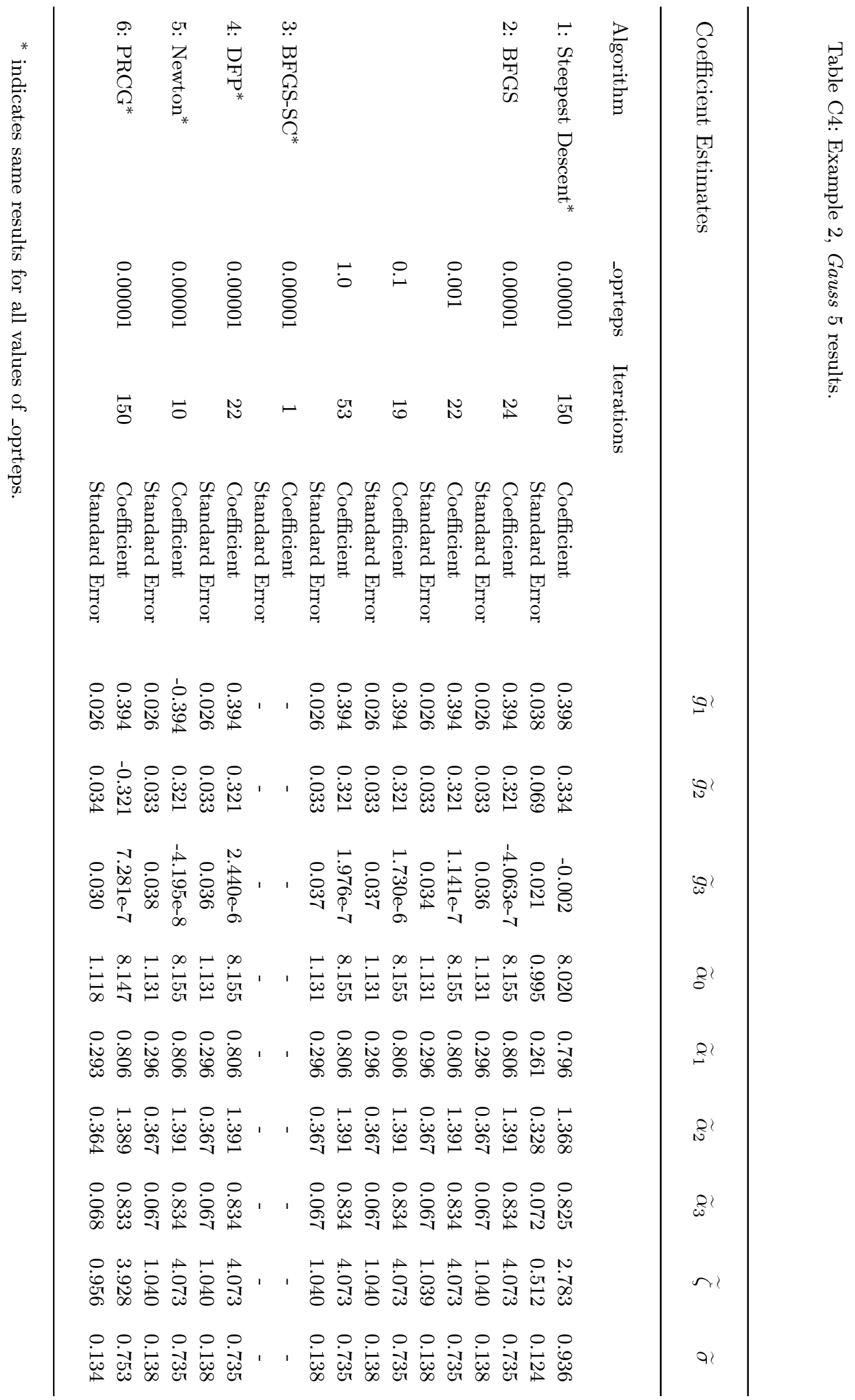

